\title{
34. THERMODYNAMIC ASPECTS OF LEG 126 INTERSTITIAL WATERS ${ }^{1}$
}

\author{
Per Kristian Egeberg ${ }^{2}$
}

\begin{abstract}
The Ocean Drilling Program Leg 126 sites may be classified into two categories depending on the presence (Group I: Sites 787, 792, and 793) or absence (Group II: Sites 788, 790, and 791) of steep concentration gradients. Shipboard X-ray diffraction analyses of bulk sediments from Group I sites revealed the presence of a number of diagenetic minerals (some of which are incompatible), but no systematic diagenetic zonation. The results of the chemical analyses of the pore waters from Group I have been used to estimate the activities of dissolved species. Thermodynamic analyses of the composition of the pore waters and the stability of authigenic minerals (gypsum, zeolites, feldspars, smectites, chlorites, and micas) show that the pore waters are close to equilibrium with most of the observed phases. Thus, only a small perturbation of the system (substitution in minerals and fluctuations in pore-water composition, in particular, in $\mathrm{pH}$ and $\mathrm{SiO}_{2}$ activity) will cause any of these phases to precipitate. Therefore, one would not expect mineralogical observations to show systematic vertical zonations at these sites. It is suggested that chlorites and high-temperature zeolites are not diagenetic sensu stricto, but were eroded from volcaniclastic highs. The absence of concentration gradients at the Group II sites has been analyzed in terms of reaction kinetics, hydrothermal advection, and temperature distribution. The absence of diagenetic imprints on the pore-water concentration profiles at these sites is probably caused by the slow nucleation of silica phases.
\end{abstract}

\section{INTRODUCTION}

Interstitial water samples extracted from sediment cores from Sites $787,788,790,791,792$, and 793 have been analyzed with respect to major and minor elements. The sites are located in the forearc basin (Sites 787, 792, and 793), on the eastern margin of the Sumisu Rift (788), and near the center of the Sumisu Rift (790 and 791) (Fig. 1). Thus, the sites cover a large range of thermal regimes, with steep thermal gradients in the backarc and more gentle gradients over the subducting plate. This report addresses two points of relevance to the island arc geological setting: (1) a thermodynamic analyses of low-temperature alteration of volcanogenic material, and (2) the effect of hydrothermal circulation on pore-water composition.

\section{ANALYTICAL METHODS}

The interstitial waters were obtained by shipboard extraction using the procedure and equipment described by Manheim and Sayles (1974). Dissolved iron in the pore waters from Sites 787, 792, and 793 was determined with furnace atomic absorption. The analytical methods for the other parameters listed in Table 1 are reported elsewhere (Taylor, Fujioka, et al., 1990). The samples for $\mathrm{Fe}^{2+}$ analyses were acidified to a $\mathrm{pH}$ of about 2 with $\mathrm{HCl}$ immediately after extraction. The extraction was not conducted under inert atmosphere conditions, but contact with air was minimized. The same procedure has been used for more than $15 \mathrm{yr}$ by the Deep Sea Drilling Project (DSDP) and the Ocean Drilling Program (ODP), and no problem with oxidation of $\mathrm{Fe}^{2+}$ has been reported. Although individual samples may be accidentally oxidized, there is no reason to doubt the general trend and level of $\mathrm{Fe}^{2+}$ given in Table 1 .

The activities of the dissolved species included in the following discussion were estimated with the geochemical computer code SOLMINEQ.88 (Kharaka et al., 1988) using the Pitzer formulations. The calculations (for Sites 787, 792, and 793) were conducted at in situ temperature using the geothermal gradient determined at Site 792 $\left(54^{\circ} \mathrm{C} \bullet \mathrm{km}^{-1}\right)$ and at a fixed pressure of 300 bars (average hydrostatic

\footnotetext{
'Taylor, B., Fujioka, K., et al., 1992. Proc. ODP, Sci. Results, 126: College Station, TX (Ocean Drilling Program).

${ }^{2}$ Department of Geology, P. O. Box 1047, Blindern, N-0316 Oslo 3, Norway (present address: Agder College, P. O. Box 607, 4601 Kristiansand, Norway).
}

pressure. The effect on the estimated activities of varying the pressure according to the actual in situ pressure may be ignored). For the samples that yielded too little water to allow the $\mathrm{pH}$ to be measured (Table 1), a pH estimated by interpolation was used as input for the activity calculations.

\section{RESULTS}

The chemical composition of the interstitial waters are given in Table 1. For the purpose of the discussion, it is convenient to classify the sites into two categories: (1) sites with steep chemical gradients (787, 792, and 793) and (2) sites with only minor variations in the pore-water composition (788, 790, and 791). A brief description of the most salient features of the pore-water composition and relevant sedimentological data is given below.

\section{Sites with Steep Concentration Gradients}

This category includes Sites 787, 792, and 793, which are situated in the forearc basin (Fig. 1). Paleontological and sedimentological data suggest that the forearc basin was formed by rifting no earlier than the middle Oligocene, and that the newly formed basin had initial depths of 4-5 km. Oligocene sedimentation occurred at a rate of $250-300 \mathrm{~m} / \mathrm{m} . \mathrm{y}$. (Sites 792 and 793) and $50 \mathrm{~m} / \mathrm{m}$.y. (Site 787) and consisted of debris-flow deposits and turbidites produced by concurrent volcanism and erosion of volcaniclastics from surrounding highs. At Site 787 (which is located in the Aogashima Canyon), up to $1 \mathrm{~km}$ of sediment was eroded during late the Miocene or Pliocene. At all sites the early Miocene slow accumulation of nannofossil claystones reflects reduced arc volcanism.

The results from Sites 792 and 793 have been reported elsewhere (Egeberg et al., 1990). The pore waters in the Oligocene sections at these sites constitute a distinct type of $\mathrm{CaCl}_{2}$ brines. At Site 792, a maximum $\mathrm{Ca}^{2+}$ concentration of $169.3 \mathrm{mM}$ is observed at $599 \mathrm{mbsf}$ (meters below sea floor); and at Site 793, the $\mathrm{Ca}^{2+}$ concentration reaches a stable level of $300 \mathrm{mM}$ below $997 \mathrm{mbsf}$. The concentration of $\mathrm{Na}^{+}$in this region is about $160 \mathrm{mM}$ (Fig. 2). The low concentration of $\mathrm{Mg}^{2+}$ (Table 1) shows that the reversal of the concentration profiles of $\mathrm{Na}^{+}$and $\mathrm{Ca}^{2+}$ toward the basement at Site 792 cannot be the result of contamination by seawater during drilling. At both sites ( 792 and 793), concentrations of $\mathrm{K}^{+}$and $\mathrm{Mg}^{2+}$ drop toward zero across the Miocene/Oligocene boundary (Table 1), and the concentration of 

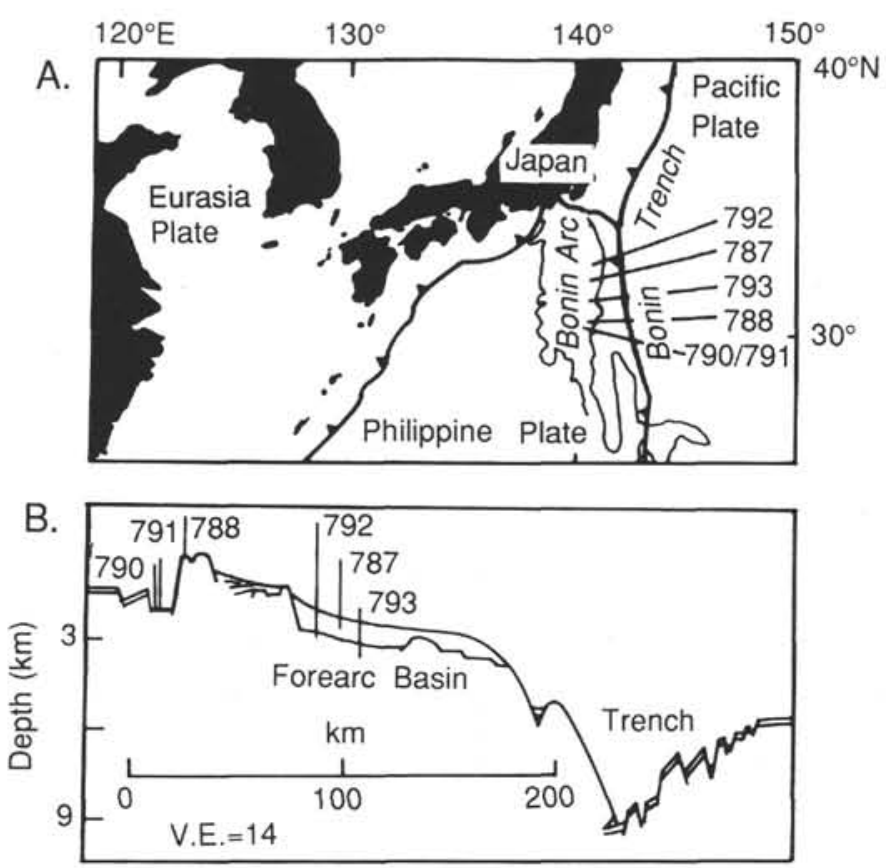

Figure 1. A. Location of the sites in the Pacific Ocean. B. Projection of Leg 126 sites onto a schematic section across the Izu-Bonin Arc. V.E. = vertical exaggeration.

$\mathrm{SO}_{4}^{2}$ - decreases by about $50 \%$. At Site 793 a significant increase in $\mathrm{Cl}^{-}$is observed across the Miocene/Oligocene boundary, and a stable level of about $700-720 \mathrm{mM}$ is reached below 997 mbsf. This is attributed to uptake of water into hydrous diagenetic minerals (zeolites, gypsum, and smectites; Egeberg et al., 1990). Moreover, porewater titration alkalinities decrease from $2-4 \mathrm{mM}$ to about $0.5 \mathrm{mM}$ in the Oligocene sections, $\mathrm{pH}$ increases by about $1 \mathrm{pH}$ unit from 7.7 in the Miocene sections, and concentrations of dissolved silica drop pronouncedly (from $730 \mu \mathrm{M}$ to $216 \mu \mathrm{M}$ ) in the Oligocene sections (average for both sites).

The pore waters from Site 787 show all the features of the above sites, but they exhibit less pronounced variations, and the gradients are positioned at much shallower depths (Fig. 2). This is probably related to the fact that about $1 \mathrm{~km}$ of the section has been eroded at this site. Concentrations of $\mathrm{Ca}^{2+}$ do not increase above $80 \mathrm{mM}$, and concentrations of $\mathrm{Na}^{+}$and $\mathrm{SO}_{4}^{2-}$ are only slightly lower than in seawater of similar chlorinity. Concentrations of $\mathrm{Mg}^{2+}$ and $\mathrm{K}^{+}$decrease with increasing depth, but they do not reach as low levels as at Sites 792 and 793. Low concentrations of $\mathrm{Na}^{+}$and $\mathrm{Cl}^{-}$in the sample from Section 126-787B-12R-1 (Table 1) reflect some mixing with drilling mud filtrate. The reversal of the $\mathrm{Ca}^{2+}, \mathrm{Mg}^{2+}$, and $\mathrm{K}^{+}$profiles in the deepest sample is most probably a result of mixing with seawater during drilling.

\section{Sites with Weak Concentration Gradients}

This category includes Sites 788, 790, and 791 situated on the eastern margin of the Sumisu Rift (788) and near the center of the Sumisu Rift (790 and 791) (Fig. 1). At Site 788, the section covered by the pore-water data (8.45-214.65 mbsf) consists of pumiceous sandy granule and pebble gravel locally interbedded with sand and rare vitric silts. The sedimentary sections at Site 791 are about three times thicker than the time-equivalent sections at Site 790. However, although the sediments are coarser at Site 791 , the lithotypes and stratigraphy at the two sites are similar. At both sites two units were identified. At Site 791, Unit I consists of $44 \%$ pumiceous gravel, $30 \%$ pumiceous sand and vitric silt, and $12 \%$ clay and

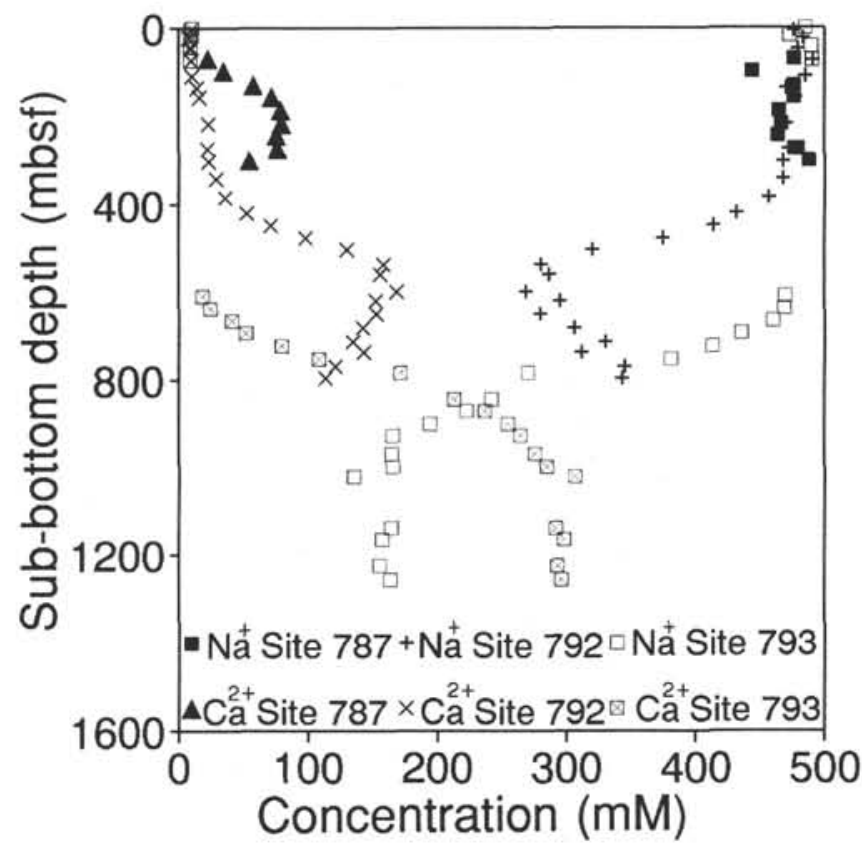

Figure 2. Concentrations of $\mathrm{Ca}^{2+}$ and $\mathrm{Na}^{+}$at Sites 787, 792, and 793.

clayey silt. About $90 \%$ of Unit II consists of nannofossil clay, carbonate-rich claystone and silty claystone, and carbonate silty claystone. The boundary between Units I and II were placed at 165 and 428.5 mbsf at Sites 790 and 791, respectively. Sediment accumulation rates at each site exhibit an exponential increase over the recovered interval, with present-day values of about 1000 and $2200 \mathrm{~m} / \mathrm{m}$.y. at Sites 790 and 791 , respectively.

Concentrations of the major elements differ little from the composition of seawater. At Sites 790 and 791, the samples with low concentrations of $\mathrm{SiO}_{2}$ may be contaminated by seawater (marked with an asterisk in Table 1).

\section{DISCUSSION}

The composition of interstitial water in deep-sea sediments is controlled by three different processes: (1) reactions of pore-water constituents with components of the solid matrix, (2) diffusion of dissolved constituents, and (3) advection of pore water. Because reactive components are unevenly distributed, reactions may cause concentration extremes. Diffusion will smooth concentration extremes; advection will tend to displace them and, if sufficiently rapid compared with the rate of reaction, eliminate concentration gradients. Thus, the absence of steep concentration gradients may signify either low rates of reaction relative to diffusion, a short life time of the system, or high rates of advection. The pore-water profiles determined in this study exhibit both steep and gentle gradients, suggesting that they represent reaction-dominated and advection-dominated systems, respectively. Altematively, the absence of steep concentration gradients may be due to slow rates of reaction. These sites will be discussed separately.

\section{Sites with Steep Concentration Gradients}

Finely dispersed volcanic material is highly unstable and decomposes more readily than nearly all associated phases. Thus, there is little doubt that the highly altered composition of the pore waters at Sites 787,792 , and 793 is caused by the low-temperature alteration of volcanic material. The fact that the pore-water gradients coincide with the Miocene/Oligocene boundaries that define the transition 
between hemipelagic sedimentation and volcanogenic debris-flow deposits indicates that the rate of the vertical flow of pore water is small compared with the rate of the governing reactions.

One very interesting aspect of the concentration profiles is that they reach more or less stable levels in the Oligocene volcanogenic sections (Sites 787 and 793). Thus, there must be sinks for $\mathrm{Na}^{+}, \mathrm{Ca}^{2+}$, $\mathrm{Mg}^{2+}, \mathrm{Fe}^{2+}, \mathrm{SiO}_{2}$, and $\mathrm{K}^{+}$released from the volcaniclastics. The aim of this section is to present which phases thermodynamics predict as potential sinks.

The concurrent low $\mathrm{SO}_{4}^{2-}$ and high $\mathrm{Ca}^{2+}$ concentrations in the Oligocene sections at Sites 792 and 793 suggest precipitation of gypsum. Because of the very low concentration of organic matter ( $<0.03 \%$ organic carbon), bacterial sulfate reduction cannot have depleted the sulfate. At both sites gypsum was detected by X-ray diffraction (XRD) analyses. Figure 3 compares the ion activity product with the solubility of gypsum.

At all sites the pore waters are modeled to have reached equilibrium with respect to gypsum at depth in the Oligocene sections (Fig. 3). The reduction of sulfate corresponds to the formation of about $0.1 \%$ gypsum (volume of solid fraction). However, significantly more $\mathrm{Ca}^{2+}$ has been released from volcanic debris than can be accounted for by the sum of gypsum and pore-water buildup of $\mathrm{Ca}^{2+}$ (Egeberg et al., 1990). Hence, although the precipitation of gypsum controls the activity of $\mathrm{SO}_{4}^{2-}$, gypsum is not the major sink of $\mathrm{Ca}^{2+}$.

Zeolites are ubiquitous alteration products in tuffs and are probably the major sinks for $\mathrm{Ca}^{2+}$. Table 2 shows the zeolites identified by shipboard XRD analyses (Taylor, Fujioka, et al., 1990) and the composition of their $\mathrm{Ca}^{2+}, \mathrm{Na}^{+}$, and $\mathrm{K}^{+}$end members. Other identified minerals that may be of authigenic origin include feldspars, illites, smectites, and chlorites. Because the Oligocene sections were deposited by debris flow and turbidites, it is quite possible that some of these minerals were formed elsewhere and thus cannot be regarded as sinks for elements released during in situ alteration of volcanic material. A thermodynamic analyses may help to resolve this question.

The large compositional variations of zeolites and the low temperatures of the diagenetic environment represent the major uncertainty in this kind of analyses. Thus, the results may only be used as general guidelines for a discussion of diagenetic alterations under marine conditions in practically closed systems (Egeberg et al., 1990).

Low-grade metamorphic zeolite assemblages have been subjected to numerous studies (e.g., Coombs, 1954; Surdam and Boles, 1979; Boles, 1981a). Fewer studies, however, deal with the low-temperature diagenetic environment of the seafloor and the first few hundred meters of burial (e.g., Stonecipher, 1977; Boles, 1981b). Only a few authors (e.g., Cosgrove and Papavassiliou, 1979) have compared the zeolite assemblage and chemistry with the composition of pore waters of marine origin. This may be caused partly by the scarcity of thermodynamic data and also by the lack of analyses of the water associated with the zeolites. The thermodynamic data used here were estimated by the algorithm of Iglesia and Aznar (1986) and by data and regression equations from Helgeson et al. (1978).

The thermodynamic parameters of the minerals change significantly over the temperature interval covered by the samples. Unfortunately the thermal gradient could only be measured at Site 792 $\left(54^{\circ} \mathrm{C} \cdot \mathrm{km}^{-1}\right)$. As a first approximation the same thermal gradient was used at all sites $(787,792$, and 793$)$.

A comparison of Figures 2 and 3 shows that the intervals characterized by approximately constant pore-water composition and gypsum saturation cover the temperature intervals $10^{\circ}-20^{\circ} \mathrm{C}$ (Site 787), $30^{\circ}-50^{\circ} \mathrm{C}$ (Site 792 ) and $50^{\circ}-70^{\circ} \mathrm{C}$ (Site 793). The stability diagrams below were constructed at $15^{\circ}, 40^{\circ}$, and $60^{\circ} \mathrm{C}$ for Sites 787,792 , and 793 , respectively.

One of the few statements about the distribution of zeolites in deep-sea sediments that seems to have gained general acceptance is that, with increasing sediment age, the abundance of clinoptilolites increases relative to the abundance of phillipsite (e.g., Boles, 1981b) and that phillipsite is a metastable phase in the deep sea environment. At fixed pressure (P), temperature $(\mathrm{T})$, and composition, the relative stability of phillipsite and clinoptilolite depends only on the activity of dissolved silica (Fig. 4). At Sites 787 and 792, clinoptilolite is stable relative to phillipsite. At Site 793 there is an apparent shift toward phillipsite stability. However, in accordance with the statement above and other thermodynamic analyses presented below, both clinoptilolites and phillipsites are unstable at Site 793. Thus, it should not be inferred that phillipsite can form at the expense of clinoptilolite.

To increase the readability, the multi-component diagrams will be discussed separately. A brief summary is included at the end of this section.

\section{FeO-MgO- $\mathrm{Al}_{2} \mathrm{O}_{3}-\mathrm{SiO}_{2}-\mathrm{H}_{2} \mathrm{O}$}

The alteration of volcanogenic material releases significant amounts of $\mathrm{Mg}^{2+}$ and $\mathrm{Fe}^{2+}$, yet the pore-water concentration of these elements is very low $\left(\mathrm{Fe}^{2+}\right)$ or decreases with increasing depth $\left(\mathrm{Mg}^{2+}\right)$. Chlorites, smectites, and carbonates are likely sinks for these elements. The Shipboard Scientific Party (Taylor, Fujioka, et al., 1990) found that chlorites are very scarce at Site 792. At Site 793, chlorites are present in the depth interval 1.3-74.0 mbsf and in a few samples below this depth. Chlorites are common between 839.7 and $883.7 \mathrm{mbsf}$. The clay mineral assemblage in the Oligocene sections is overwhelmingly dominated by trioctahedral smectites. Dolomite and ankerites are rare, and siderite was not detected. This suggests that smectite is the dominant sink of $\mathrm{Fe}^{2+}$ and $\mathrm{Mg}^{2+}$. The amount of $\mathrm{Mg}^{2+}$ and $\mathrm{Fe}^{2+}$ in the unmodified pore water (seawater) is negligible compared with the amount released during alteration of the volcanic matter. Therefore, the $\mathrm{Mg} / \mathrm{Fe}$ ratio of the smectite will approach that of the bulk sediment $(\mathrm{Mg} / \mathrm{Fe}=5.7$; Taylor, Fujioka, et al., 1990). A saponite satisfying this condition may be represented by the formula:

$$
\mathrm{Na}_{0.33}\left(\mathrm{Mg}_{2.55} \mathrm{Fe}_{0.45}\right)\left(\mathrm{Al}_{0.33} \mathrm{Si}_{3.67}\right) \mathrm{O}_{10}(\mathrm{OH})_{2} \text {. }
$$

To illustrate the uncertainty introduced by the stoichiometry of the smectite, phase boundaries were also constructed for a saponite with a $\mathrm{Mg} / \mathrm{Fe}$ ratio of 2.6 (MacEvan and Wilson, 1980):

$$
\mathrm{Na}_{0.33}\left(\mathrm{Mg}_{2.167} \mathrm{Fe}_{0.833}\right)\left(\mathrm{Al}_{0.33} \mathrm{Si}_{3.67}\right) \mathrm{O}_{10}(\mathrm{OH})_{2} \text {. }
$$

The thermodynamic parameters for the trioctahedral smectites were estimated by means of data and algorithms taken from Wolery (1979) and Helgeson et al. (1978).

The relative stability of chlorites (data from Walshe, 1986), dioctahedral smectites (represented by pyrophyllite; Aagaard and Helgeson, 1983), and trioctahedral smectites is compared in Figures 5A-5C. It is seen that there is a systematic evolution of the pore waters from mixed di/trioctahedral smectite stability at low temperatures (Site 787) toward increasing trioctahedral smectite stability at higher temperatures. At no point does the pore-water composition plot close to the stability field of the chlorites. Note that these observations are independent of which of the two trioctahedral smectites one chooses to consider. The apparent instability of the chlorites raises a question about their origin. Because the relative stability of chlorites over trioctahedral smectites increases rapidly with decreasing aqueous $\mathrm{SiO}_{2}$ activity, the chlorites may have formed during transient periods when the $\mathrm{SiO}_{2}$ activity was lowered by precipitation of other authigenic minerals. However, the Oligocene section was deposited by debris flow and turbidites and the possibility that the chlorites have been formed elsewhere cannot be excluded.

\section{$\mathrm{Na}_{2} \mathrm{O}-\mathrm{Al}_{2} \mathrm{O}_{3}-\mathrm{SiO}_{2}-\mathrm{H}_{2} \mathrm{O}$}

Hydrolysis of the andesitic sediments releases $\mathrm{Na}^{+}$to the pore water. Thus, the stable $\mathrm{Na}^{+}$level at Site 787 and the decreasing $\mathrm{Na}^{+}$concentrations at Sites 792 and 793 (Fig. 1) require precipitation of Na containing secondary minerals. The considerable variations in $\mathrm{Na}^{+}$suggest that there may be a sequence of diagenetic mineral precipitation.

Figures $6 \mathrm{~A}-6 \mathrm{C}$ show that the size of the stability field of dioctahedral smectites is strongly reduced by the presence of stilbite. This shows that $\mathrm{Na}$-rich dioctahedral smectites are unstable at all temperatures and cannot 
Table 1. Pore-water composition at Sites 787, 788, 790, 791, 792, and 793.

\begin{tabular}{|c|c|c|c|c|c|c|c|c|c|c|c|c|}
\hline $\begin{array}{l}\text { Core, section, } \\
\text { interval }(\mathrm{cm})\end{array}$ & $\begin{array}{l}\text { Depth } \\
\text { (mbsf) }\end{array}$ & $\begin{array}{l}\text { Univ } \\
\text { subunit }\end{array}$ & $\mathrm{pH}$ & $\begin{array}{c}\text { Alkalinity } \\
\text { (meq/) }\end{array}$ & $\underset{(\mathrm{mM})}{\mathrm{Cr}^{2}}$ & $\begin{array}{l}\mathrm{SO}_{4}^{2-} \\
(\mathrm{mM})\end{array}$ & $\begin{array}{l}\mathrm{Na}^{+} \\
(\mathrm{mM})\end{array}$ & $\begin{array}{l}\mathrm{Mg}^{2+} \\
(\mathrm{mM})\end{array}$ & $\begin{array}{l}\mathrm{Ca}^{2+} \\
(\mathrm{mM})\end{array}$ & $\underset{(\mathrm{mM})}{\mathrm{K}^{+}}$ & $\begin{array}{l}\mathrm{Fe}^{2+} \\
(\mu \mathrm{M})\end{array}$ & $\begin{array}{l}\mathrm{SiO}_{2} \\
(\mu \mathrm{M})\end{array}$ \\
\hline $\begin{array}{l}126-787 \mathrm{~B}- \\
9 \mathrm{R}-2,65-75 \\
12 \mathrm{R}-1,3-13 \\
15 \mathrm{R}-2,146-156 \\
18 \mathrm{R}-1,80-90 \\
21 \mathrm{R}-2,140-150 \\
24 \mathrm{R}-2,140-150 \\
27 \mathrm{R}-2,42-52 \\
30 \mathrm{R}-2,140-150 \\
33 \mathrm{R}-1,58-68\end{array}$ & $\begin{array}{r}71.20 \\
98.18 \\
130.21 \\
157.05 \\
187.95 \\
217.05 \\
244.97 \\
274.95 \\
301.43\end{array}$ & $\begin{array}{l}\text { III } \\
\text { III } \\
\text { IVA } \\
\text { IVA } \\
\text { IVA } \\
\text { IVA } \\
\text { IVA } \\
\text { IVA } \\
\text { IVB }\end{array}$ & $\begin{array}{l}7.43 \\
7.16 \\
8.07 \\
8.54 \\
7.04 \\
8.67 \\
8.55\end{array}$ & $\begin{array}{l}1.52 \\
1.09 \\
0.99 \\
0.62 \\
0.76 \\
0.37\end{array}$ & $\begin{array}{l}561 \\
542 \\
562 \\
582 \\
572 \\
590 \\
578 \\
586 \\
575\end{array}$ & $\begin{array}{l}28.4 \\
27.6 \\
23.9 \\
27.8 \\
25.5 \\
26.3 \\
27.2 \\
29.7 \\
30.7\end{array}$ & $\begin{array}{l}476 \\
444 \\
476 \\
476 \\
465 \\
467 \\
464 \\
480 \\
488\end{array}$ & $\begin{array}{r}44.5 \\
37.9 \\
7.2 \\
4.3 \\
1.3 \\
3.5 \\
1.8 \\
2.6 \\
15.5\end{array}$ & $\begin{array}{l}23.4 \\
35.3 \\
57.8 \\
72.5 \\
79.0 \\
79.8 \\
76.1 \\
76.6 \\
55.1\end{array}$ & $\begin{array}{l}7.1 \\
3.9 \\
3.0 \\
2.1 \\
1.9 \\
2.0 \\
2.2 \\
2.7 \\
4.2\end{array}$ & $\begin{array}{l}0.3 \\
2.8 \\
0.5 \\
0.2 \\
0.2 \\
0.2 \\
0.2\end{array}$ & $\begin{array}{r}843 \\
559 \\
68 \\
94 \\
354 \\
581 \\
485 \\
528 \\
516\end{array}$ \\
\hline $\begin{array}{l}126-788 \mathrm{C}- \\
1 \mathrm{H}-3,140-150 \\
3 \mathrm{H}-5,140-150 \\
7 \mathrm{H}-1,140-150 \\
9 \mathrm{H}-3,140-150 \\
13 \mathrm{H}-6,0-5 \\
16 \mathrm{H}-1,140-150 \\
23 \mathrm{H}-3,140-150\end{array}$ & $\begin{array}{r}8.45 \\
27.65 \\
59.65 \\
81.65 \\
122.75 \\
145.15 \\
214.65\end{array}$ & $\begin{array}{l}\text { IA } \\
\text { IA } \\
\text { IA } \\
\text { IA } \\
\text { IA } \\
\text { IA } \\
\text { IA }\end{array}$ & $\begin{array}{l}7.27 \\
7.44 \\
7.49 \\
7.51 \\
8.03 \\
8.09 \\
7.97\end{array}$ & $\begin{array}{l}2.31 \\
2.36 \\
2.38 \\
2.48 \\
2.53 \\
2.59 \\
2.50\end{array}$ & $\begin{array}{l}557 \\
554 \\
552 \\
557 \\
556 \\
556 \\
556\end{array}$ & $\begin{array}{l}28.3 \\
29.7 \\
28.9 \\
28.8 \\
29.6 \\
27.9 \\
28.3\end{array}$ & $\begin{array}{l}477 \\
480 \\
475 \\
481 \\
477 \\
469 \\
476\end{array}$ & $\begin{array}{l}51.9 \\
51.8 \\
51.9 \\
52.2 \\
52.1 \\
52.6 \\
52.5\end{array}$ & $\begin{array}{l}11.2 \\
11.2 \\
11.1 \\
11.5 \\
11.4 \\
11.5 \\
11.4\end{array}$ & $\begin{array}{l}11.3 \\
11.0 \\
11.3 \\
11.2 \\
10.9 \\
11.0 \\
11.3\end{array}$ & $\begin{array}{l}3.6 \\
2.2 \\
3.8 \\
0.6 \\
1.2 \\
2.3 \\
4.8\end{array}$ & $\begin{array}{r}497 \\
459 \\
440 \\
518 \\
58 \\
537 \\
495\end{array}$ \\
\hline $\begin{array}{l}126-790 \mathrm{~A}- \\
2 \mathrm{H}-3,140-150 \\
3 \mathrm{H}-5,140-150\end{array}$ & $\begin{array}{l}13.25 \\
25.75\end{array}$ & $\begin{array}{l}\text { I } \\
\text { I }\end{array}$ & $\begin{array}{l}7.66 \\
7.62\end{array}$ & $\begin{array}{l}4.46 \\
3.94\end{array}$ & $\begin{array}{l}554 \\
553\end{array}$ & $\begin{array}{l}27.9 \\
27.4\end{array}$ & $\begin{array}{l}474 \\
470\end{array}$ & $\begin{array}{l}53.5 \\
53.1\end{array}$ & $\begin{array}{r}9.7 \\
10.2\end{array}$ & $\begin{array}{r}10.2 \\
9.3\end{array}$ & $\begin{array}{l}9.0 \\
7.0\end{array}$ & $\begin{array}{l}677 \\
477\end{array}$ \\
\hline $\begin{array}{l}126-790 \mathrm{~B}- \\
\text { \% } 6 \mathrm{H}-3,140-150 \\
8 \mathrm{H}-5,140-150 \\
{ }^{9} 9 \mathrm{H}-2,140-150\end{array}$ & $\begin{array}{l}46.95 \\
69.05 \\
74.25\end{array}$ & $\begin{array}{l}\text { I } \\
\text { I } \\
\text { I }\end{array}$ & $\begin{array}{l}17.93 \\
7.64 \\
17.56\end{array}$ & $\begin{array}{l}2.70 \\
4.79 \\
4.90\end{array}$ & $\begin{array}{l}555 \\
560 \\
561\end{array}$ & $\begin{array}{l}27.8 \\
27.6 \\
27.2\end{array}$ & $\begin{array}{l}478 \\
475 \\
485\end{array}$ & $\begin{array}{l}53.0 \\
52.2 \\
52.6\end{array}$ & $\begin{array}{r}10.8 \\
9.6 \\
10.3\end{array}$ & $\begin{array}{l}9.2 \\
8.0 \\
6.5\end{array}$ & $\begin{array}{r}7.0 \\
12.0 \\
7.0\end{array}$ & $\begin{array}{l}239 \\
823 \\
500\end{array}$ \\
\hline $\begin{array}{l}126-790 \mathrm{C}- \\
2 \mathrm{H}-4,140-150 \\
5 \mathrm{H}-5,140-150 \\
10 \mathrm{X}-1,101-111 \\
13 \mathrm{X}-2,140-150 \\
16 \mathrm{X}-3,140-150 \\
18 \mathrm{X}-2,140-150\end{array}$ & $\begin{array}{l}100.45 \\
130.95 \\
165.96 \\
196.85 \\
227.25 \\
254.65\end{array}$ & $\begin{array}{l}\text { I } \\
\text { I } \\
\text { II } \\
\text { II } \\
\text { II } \\
\text { II }\end{array}$ & $\begin{array}{l}7.69 \\
7.62 \\
7.45 \\
7.36 \\
7.39 \\
7.40\end{array}$ & $\begin{array}{l}3.99 \\
3.40 \\
5.68 \\
9.29 \\
9.40 \\
5.99\end{array}$ & $\begin{array}{l}563 \\
567 \\
563 \\
560 \\
560 \\
560\end{array}$ & $\begin{array}{l}26.8 \\
25.3 \\
21.2 \\
15.1 \\
18.2 \\
22.9\end{array}$ & $\begin{array}{l}483 \\
478 \\
472 \\
472 \\
473 \\
478\end{array}$ & $\begin{array}{l}53.8 \\
51.9 \\
52.3 \\
52.0 \\
51.9 \\
50.0\end{array}$ & $\begin{array}{r}11.7 \\
11.0 \\
9.5 \\
5.6 \\
5.7 \\
7.5\end{array}$ & $\begin{array}{l}5.4 \\
6.7 \\
7.1 \\
8.1 \\
8.7 \\
9.9\end{array}$ & $\begin{array}{r}43.0 \\
12.0 \\
6.0 \\
11.0 \\
11.0 \\
16.0\end{array}$ & $\begin{array}{r}392 \\
718 \\
956 \\
962 \\
942 \\
1003\end{array}$ \\
\hline $\begin{array}{l}126-791 \mathrm{~A}- \\
2 \mathrm{H}-5,140-150 \\
5 \mathrm{H}-3,140-150 \\
\mathrm{a}^{9} \mathrm{H}-6,140-150 \\
=12 \mathrm{H}-5,140-150 \\
=15 \mathrm{H}-6,25-35 \\
18 \mathrm{H}-6,90-100 \\
22 \mathrm{H}-6,140-150\end{array}$ & $\begin{array}{r}11.95 \\
37.45 \\
80.25 \\
107.85 \\
137.30 \\
166.95 \\
205.85\end{array}$ & $\begin{array}{l}\text { I } \\
\text { I } \\
\text { I } \\
\text { I } \\
\text { I } \\
\text { I } \\
\text { I }\end{array}$ & $\begin{array}{l}7.47 \\
7.56 \\
7.86 \\
7.77 \\
7.82 \\
7.77 \\
7.53\end{array}$ & $\begin{array}{l}4.11 \\
3.01 \\
3.19 \\
3.54 \\
3.72 \\
4.66 \\
4.55\end{array}$ & $\begin{array}{l}569 \\
576 \\
563 \\
558 \\
565 \\
570 \\
571\end{array}$ & $\begin{array}{l}28.5 \\
27.5 \\
28.1 \\
28.8 \\
26.6 \\
26.9 \\
26.5\end{array}$ & $\begin{array}{l}487 \\
493 \\
478 \\
473 \\
478 \\
484 \\
486\end{array}$ & $\begin{array}{l}45.8 \\
44.3 \\
52.8 \\
52.1 \\
52.8 \\
53.0 \\
52.7\end{array}$ & $\begin{array}{l}19.2 \\
19.7 \\
12.0 \\
12.3 \\
10.9 \\
10.8 \\
10.8\end{array}$ & $\begin{array}{l}6.8 \\
7.3 \\
9.5 \\
8.8 \\
8.3 \\
6.6 \\
6.6\end{array}$ & $\begin{array}{l}88.0 \\
35.0 \\
\text { LD } \\
\text { LD } \\
4.0 \\
\text { LD } \\
1.0\end{array}$ & $\begin{array}{l}284 \\
576 \\
269 \\
338 \\
263 \\
506 \\
487\end{array}$ \\
\hline $\begin{array}{l}126-791 \mathrm{~B}- \\
10 \mathrm{R}-1,86-96 \\
13 \mathrm{R}-1,140-150 \\
23 \mathrm{R}-3,115-125 \\
28 \mathrm{R}-3,140-150 \\
34 \mathrm{R}-3,140-150 \\
39 \mathrm{R}-1,140-150 \\
42 \mathrm{R}-2,0-10 \\
45 \mathrm{R}-2,140-150\end{array}$ & $\begin{array}{l}474.21 \\
503.55 \\
602.90 \\
651.05 \\
709.05 \\
753.85 \\
782.85 \\
813.20\end{array}$ & $\begin{array}{l}\text { II } \\
\text { II } \\
\text { II } \\
\text { II } \\
\text { II } \\
\text { II } \\
\text { II } \\
\text { II }\end{array}$ & $\begin{array}{l}7.53 \\
7.48 \\
7.33 \\
7.34 \\
7.39 \\
7.45 \\
7.32 \\
7.48\end{array}$ & $\begin{array}{l}3.76 \\
2.39 \\
4.58 \\
3.98 \\
3.77 \\
4.10 \\
3.33 \\
2.32\end{array}$ & $\begin{array}{l}557 \\
566 \\
561 \\
562 \\
558 \\
561 \\
562 \\
558\end{array}$ & $\begin{array}{l}27.6 \\
26.8 \\
25.5 \\
24.2 \\
25.9 \\
26.2 \\
26.1 \\
26.7\end{array}$ & $\begin{array}{l}484 \\
483 \\
481 \\
491 \\
469 \\
481 \\
480 \\
483\end{array}$ & $\begin{array}{l}48.7 \\
49.5 \\
49.5 \\
47.9 \\
49.6 \\
50.4 \\
50.3 \\
49.6\end{array}$ & $\begin{array}{l}15.7 \\
16.1 \\
15.7 \\
14.2 \\
13.1 \\
13.0 \\
13.2 \\
12.5\end{array}$ & $\begin{array}{r}5.8 \\
5.6 \\
5.7 \\
6.3 \\
9.6 \\
9.7 \\
10.2 \\
10.6\end{array}$ & $\begin{array}{r}2.0 \\
3.0 \\
\mathrm{LD} \\
2.0 \\
2.0 \\
13.0 \\
3.0 \\
1.0\end{array}$ & $\begin{array}{r}717 \\
850 \\
1001 \\
1049 \\
947 \\
856 \\
999 \\
682\end{array}$ \\
\hline $\begin{array}{l}126-792 \mathrm{~A}- \\
1 \mathrm{H}-5,140-150 \\
3 \mathrm{H}-5,140-150 \\
6 \mathrm{H}-2,140-150 \\
9 \mathrm{H}-1,89-97\end{array}$ & $\begin{array}{r}7.45 \\
26.55 \\
50.15 \\
76.54\end{array}$ & $\begin{array}{l}\text { I } \\
\text { I } \\
1 \\
\text { I }\end{array}$ & $\begin{array}{l}7.74 \\
7.80 \\
7.80 \\
7.82\end{array}$ & $\begin{array}{l}3.18 \\
4.27 \\
3.33 \\
2.66\end{array}$ & $\begin{array}{l}550 \\
556 \\
561 \\
562\end{array}$ & $\begin{array}{l}28.4 \\
25.5 \\
26.3 \\
26.9\end{array}$ & $\begin{array}{l}476 \\
484 \\
480 \\
491\end{array}$ & $\begin{array}{l}52.2 \\
52.5 \\
53.0 \\
52.0\end{array}$ & $\begin{array}{r}9.4 \\
7.9 \\
9.2 \\
10.2\end{array}$ & $\begin{array}{r}10.5 \\
8.9 \\
9.3 \\
9.5\end{array}$ & $\begin{array}{l}24.3 \\
22.7 \\
19.3 \\
26.3\end{array}$ & $\begin{array}{l}521 \\
629 \\
595 \\
650\end{array}$ \\
\hline
\end{tabular}

act as sinks of $\mathrm{Na}^{+}$. The evolution of the pore waters suggests a shift from dominantly clinoptilolite stability at temperatures below $20^{\circ} \mathrm{C}$ through a zone of mixed stilbite/clinoptilolite at $30^{\circ}-50^{\circ} \mathrm{C}$ to stilbite stability at temperatures $>50^{\circ} \mathrm{C}$. This may be in agreement with the data from Stonecipher (1977, fig. 9, p. 109), which show a maximum of clinoptilolite abundance in deep-sea sediments buried at 750-800 mbsf. On the other hand, Boles (1981b) suggested that the age of the sediment is a much more critical factor than temperature. Most authors list stilbite together with the less common zeolites. Thus, the evolution of the pore waters toward stilbite stability may reflect the very special conditions at Sites 792 and 793. Note, however, that thermodynamics cannot predict the quantity of minerals.

Perhaps the most interesting feature of the stability diagrams is that at intermediate temperatures the composition of the pore waters plot close to the three phases analcime, clinoptilolite, and stilbite. This shows that only a small perturbation of the system (substitution in minerals and fluctuations in pore-water composition, in particular, in $\mathrm{pH}$ and $\mathrm{SiO}_{2}$ activity) will cause any of these phases to precipitate. Therefore, one would not expect mineralogi- cal observations to show systematic vertical Na-zeolite zonations at thesesites. This is confirmed by the shipboardXRDanalyses(Taylor, Fujioka, et al., 1990). The heterogeneity of the zeolite assemblage is enhanced by the fact that each sediment layer has experienced all temperatures between that at the seaflooranditspresenttemperature. Because the rate of dissolution of authigenic aluminosilicates is low (they are closer to equilibrium than detrital phases), phases formed at low temperatures may exist unstably for significant periods of time.

The diagrams also show that the pore waters are close to equilibrium with respect to albite. Albite is a common diagenetic constituent of sandstones rich in rhyolitic and dacitic material (Surdam and Boles, 1979). The sediments at Sites 792 and 793 are rich in feldspar. However, it may be of magmatic origin.

\section{$\mathrm{CaO}-\mathrm{Al}_{2} \mathrm{O}_{3}-\mathrm{SiO}_{2}-\mathrm{H}_{2} \mathrm{O}$}

Mass balance estimates (Egeberg et al., 1990) demonstrate that, although the pore waters are strongly enriched in $\mathrm{Ca}^{2+}$, this can only account for a small proportion of the $\mathrm{Ca}^{2+}$ released by hydrolysis of 
Table 1 (continued).

\begin{tabular}{|c|c|c|c|c|c|c|c|c|c|c|c|c|}
\hline $\begin{array}{l}\text { Core, section, } \\
\text { interval }(\mathrm{cm})\end{array}$ & $\begin{array}{l}\text { Depth } \\
\text { (mbsf) }\end{array}$ & $\begin{array}{c}\text { Unit } \\
\text { subunit }\end{array}$ & $\mathrm{pH}$ & $\begin{array}{l}\text { Alkalinity } \\
\text { (meq/) }\end{array}$ & $\underset{(\mathrm{mM})}{\mathrm{Cl}^{-}}$ & $\begin{array}{l}\mathrm{SO}_{4}^{2-} \\
(\mathrm{mM})\end{array}$ & $\begin{array}{c}\mathrm{Na}^{+} \\
(\mathrm{mM})\end{array}$ & $\begin{array}{l}\mathrm{Mg}^{2+} \\
(\mathrm{mM})\end{array}$ & $\begin{array}{l}\mathrm{Ca}^{2+} \\
(\mathrm{mM})\end{array}$ & $\underset{(\mathrm{mM})}{\mathrm{K}^{+}}$ & $\begin{array}{l}\mathrm{Fe}^{2+} \\
(\mu \mathrm{M})\end{array}$ & $\begin{array}{l}\mathrm{SiO}_{2} \\
(\mu \mathrm{M})\end{array}$ \\
\hline \multicolumn{13}{|l|}{$126-792 \mathrm{~B}-$} \\
\hline $8 X-3,140-150$ & 112.35 & I & 7.60 & 3.09 & 561 & 27.6 & 485 & 51.8 & 11.0 & 8.8 & 28.8 & 710 \\
\hline $11 X-1,75-85$ & 137.60 & I & 7.69 & 2.64 & 560 & 27.1 & 471 & 50.6 & 15.1 & 9.1 & 9.8 & 794 \\
\hline \multicolumn{13}{|l|}{$126-792 \mathrm{E}-$} \\
\hline $3 \mathrm{R}-2,60-70$ & 156.95 & I & 7.52 & 2.15 & 557 & 27.2 & 478 & 50.2 & 16.5 & 7.9 & 59.4 & 805 \\
\hline $9 \mathrm{R}-4,40-50$ & 217.55 & II & 7.69 & 0.23 & 560 & 27.8 & 471 & 46.0 & 23.5 & 5.9 & 59.8 & 765 \\
\hline $15 \mathrm{R}-2,115-125$ & 273.20 & II & 7.93 & 1.62 & 559 & 28.5 & 472 & 46.6 & 23.0 & 6.2 & 55.5 & 875 \\
\hline $18 \mathrm{R}-3,115-125$ & 303.70 & II & 7.58 & 2.34 & 559 & 28.2 & 468 & 46.4 & 23.9 & 5.3 & 68.0 & 938 \\
\hline $22 \mathrm{R}-2,140-150$ & 340.85 & II & 7.53 & 1.43 & 559 & 27.2 & 468 & 43.6 & 28.8 & 4.8 & 26.5 & 979 \\
\hline $27 \mathrm{R}-1,27-37$ & 386.62 & III & 7.34 & 1.37 & 560 & 26.6 & 457 & 40.0 & 36.9 & 3.8 & 4.3 & 924 \\
\hline $30 \mathrm{R}-5,11-21$ & 421.36 & IIII & 7.79 & 1.20 & 551 & 25.5 & 432 & 31.2 & 53.7 & 2.5 & 3.2 & 157 \\
\hline $33 \mathrm{R}-2,95-105$ & 446.70 & IV & 1.19 & 1.20 & 559 & 23.2 & 414 & 22.2 & 71.5 & 2.5 & 3.2 & 135 \\
\hline $36 \mathrm{R}-4,67-77$ & 478.42 & IV & 7.94 & 0.12 & 560 & 19.7 & 375 & 120 & 98.5 & 1.8 & 6.1 & 118 \\
\hline $39 \mathrm{R}-2,115-125$ & 504.90 & IV & 7.94 & 0.12 & 562 & $\begin{array}{l}19.1 \\
16.7\end{array}$ & 321 & $\begin{array}{r}12.0 \\
4.3\end{array}$ & $\begin{array}{l}730.5 \\
130.6\end{array}$ & 1.4 & $\begin{array}{l}0.1 \\
11.3\end{array}$ & 118 \\
\hline $42 \mathrm{R}-4,140-150$ & 537.15 & IV & & & $\begin{array}{l}502 \\
578\end{array}$ & $\begin{array}{l}10.7 \\
15.2\end{array}$ & 281 & $\begin{array}{l}4.3 \\
1.3\end{array}$ & $\begin{array}{l}130.0 \\
159.0\end{array}$ & 1.1 & 11.3 & 375 \\
\hline $45 \mathrm{R}-2,0-10$ & 561.55 & IV & & & 579 & 15.6 & 287 & 3.5 & 156.1 & 1.3 & & 123 \\
\hline $48 \mathrm{R}-7,140-150$ & 599.05 & IV & 8.48 & 0.35 & 579 & 15.3 & 270 & 1.7 & 169.3 & 1.0 & 2.9 & 262 \\
\hline 51R-2, $140-150$ & 620.05 & IV & & & 577 & 15.1 & 296 & 2.4 & 153.1 & 1.6 & & 343 \\
\hline & 650.55 & IV & & & 560 & 15.2 & 280 & 2.4 & 153.1 & 0.8 & & 123 \\
\hline $57 \mathrm{R}-5,140-150$ & 682.55 & IV & 8.11 & 0.47 & 563 & 16.3 & 306 & 2.5 & 143.1 & 0.9 & 2.0 & 447 \\
\hline $60 \mathrm{R}-6,140-150$ & 712.95 & IV & & & 568 & 18.8 & 331 & 3.4 & 135.6 & 1.2 & 3.8 & 282 \\
\hline $63 \mathrm{R}-3,138-150$ & 736.94 & IV & & & 567 & 17.3 & 313 & 2.2 & 143.8 & 0.7 & & 203 \\
\hline $66 \mathrm{R}-5,139-150$ & 768.95 & IV & & & 569 & 16.8 & 346 & 5.9 & 121.7 & 1.5 & 2.7 & 146 \\
\hline $69 R-4,136-146$ & 796.51 & $\mathrm{v}$ & & & 549 & 16.2 & 344 & 6.4 & 114.2 & 0.8 & & 508 \\
\hline \multicolumn{13}{|l|}{ 126-793A- } \\
\hline $1 \mathrm{H}-2,140-150$ & 2.95 & IA & 7.78 & 2.71 & 554 & 29.5 & 486 & 53.5 & 10.7 & 9.4 & 7.9 & 250 \\
\hline $3 \mathrm{H}-3,140-150$ & 17.95 & IB & 7.74 & 2.71 & 555 & 29.5 & 473 & 52.8 & 10.4 & 10.3 & 34.7 & 589 \\
\hline $6 \mathrm{H}-1,140-150$ & 43.45 & IB & 7.77 & 3.32 & 561 & 28.2 & 490 & 52.8 & 9.5 & 10.3 & 18.6 & 623 \\
\hline $9 \mathrm{H}-4,140-150$ & 76.85 & IB & 7.85 & 3.36 & 561 & 29.1 & 491 & 52.9 & 9.8 & 9.1 & 33.8 & 478 \\
\hline \multicolumn{13}{|l|}{$126-793 \mathrm{~B}-$} \\
\hline $3 \mathrm{R}-3,130-140$ & 608.65 & III & 7.69 & 2.07 & 560 & 29.1 & 470 & 51.4 & 19.2 & 7.2 & 18.3 & 725 \\
\hline $6 \mathrm{R}-3,0-10$ & 636.35 & III & 7.40 & 1.57 & 560 & 28.0 & $\begin{array}{l}470 \\
469\end{array}$ & 46.8 & 24.7 & 6.9 & 19.3 & 890 \\
\hline $9 \mathrm{R}-2,132-142$ & 664.97 & III & 7.52 & 1.19 & 568 & 29.3 & 460 & $\begin{array}{l}40.0 \\
41.6\end{array}$ & 41.2 & 5.2 & 10.0 & 764 \\
\hline $12 \mathrm{R}-1,100-110$ & 692.15 & III & 7.65 & 1.40 & 566 & 29.0 & 436 & $\begin{array}{l}41.0 \\
37.4\end{array}$ & 52.3 & 4.5 & 3.0 & 905 \\
\hline $15 \mathrm{R}-3,117-127$ & 724.32 & III & 7.64 & $\begin{array}{l}1.40 \\
0.88\end{array}$ & $\begin{array}{l}560 \\
569\end{array}$ & 29.5 & $\begin{array}{l}430 \\
413\end{array}$ & 25.4 & 80.2 & 2.6 & 7.9 & 914 \\
\hline $18 \mathrm{R}-3,115-125$ & 753.00 & IV & 7.63 & 0.62 & 588 & 22.5 & 381 & 14.8 & 108.6 & 1.7 & 3.2 & 158 \\
\hline $21 \mathrm{R}-6,133-143$ & 786.58 & v & & 0.02 & 586 & 17.3 & 271 & 1.5 & $\begin{array}{l}100.0 \\
171.5\end{array}$ & 0.8 & 3.2 & 22 \\
\hline $27 \mathrm{R}-7,33-58$ & 844.85 & $\mathrm{v}$ & 8.41 & 0.66 & $\begin{array}{l}380 \\
643\end{array}$ & 14.2 & 242 & 0.8 & 213.2 & 0.5 & 4.5 & 172 \\
\hline $30 \mathrm{R}-4,110-135$ & 870.13 & v & $\begin{array}{l}8.41 \\
8.81\end{array}$ & $\begin{array}{l}0.00 \\
0.64\end{array}$ & $\begin{array}{l}643 \\
662\end{array}$ & $\begin{array}{l}14.2 \\
13.8\end{array}$ & 223 & $\begin{array}{l}0.8 \\
1.6\end{array}$ & 237.3 & 0.5 & 1.1 & 143 \\
\hline $33 \mathrm{R}-6,0-25$ & 900.93 & v & 9.01 & 0.48 & 677 & 13.0 & 195 & $\begin{array}{l}1.0 \\
1.0\end{array}$ & 255.2 & 0.5 & 3.0 & 196 \\
\hline $36 \mathrm{R}-3,113-138$ & 926.56 & $\mathrm{v}$ & & & 671 & 11.9 & 166 & 2.0 & 265.1 & 0.5 & 2.7 & 196 \\
\hline $40 \mathrm{R}-5,96-121$ & 968.09 & $\dot{v}$ & & & 685 & 14.8 & 165 & 0.6 & 276.3 & 0.5 & & 196 \\
\hline $43 \mathrm{R}-5,120-150$ & 997.05 & $\mathrm{v}$ & 8.83 & 0.69 & 700 & 16.3 & 166 & 1.5 & 285.1 & 0.7 & 2.3 & 216 \\
\hline $46 \mathrm{R}-3,116-141$ & 1022.99 & $\mathrm{v}$ & & & 715 & 16.6 & 136 & 0.6 & 307.5 & 0.5 & & 298 \\
\hline $58 R-4,76-106$ & 1139.41 & $\mathrm{v}$ & & & 710 & 15.7 & 165 & 0.3 & 292.2 & 0.4 & & 167 \\
\hline $61 \mathrm{R}-3,56-86$ & 1166.61 & $\mathrm{~V}$ & & & 724 & 14.4 & 158 & 0.6 & 298.7 & 0.3 & & 197 \\
\hline $67 \mathrm{R}-4,0-30$ & 1225.45 & V & & & 717 & 14.6 & 156 & 0.5 & 293.9 & 0.4 & & 138 \\
\hline $70 \mathrm{R}-7,0-30$ & 1258.95 & $\mathrm{~V}$ & & & 722 & 15.3 & 164 & 1.2 & 296.8 & 0.3 & & 196 \\
\hline
\end{tabular}

Notes: The samples marked with an asterisk are suspected to have been contaminated by seawater. Site 787: Unit III and Subunits IVA and IVB, late Oligocene. Site 788: Subunit IA, Quaternary to Pliocene. Sites 790 and 791: Units I and II, Quaternary. Site 792: Unit I, Quaternary to late Pliocene; Unit II, late to middle Miocene; Unit III, early Miocene to late Oligocene; Unit IV, late Oligocene to early Oligocene; Unit V, ?. Site 793: Subunits IA and IB, Quaternary; Unit III, early to middle Miocene; Unit IV, early Miocene; Unit V, early to late Oligocene.

the volcanogenic material. It was shown above that gypsum is not a major sink of $\mathrm{Ca}^{2+}$, and only small amounts of dolomite, ankerite, and authigenic calcite are present. This suggests that aluminosilicates are important sinks of $\mathrm{Ca}^{2+}$.

The abscissa of the $\mathrm{CaO}-\mathrm{Al}_{2} \mathrm{O}_{3}-\mathrm{SiO}_{2}-\mathrm{H}_{2} \mathrm{O}$ diagrams have been expanded to include anorthite (Figs. 7A-7C). Anorthite may be taken to represent magmatic plagioclase. Although the activity of anorthite in plagioclase is less than unity, it is evident from the diagrams that it is highly unstable at the prevailing conditions. Figures $7 \mathrm{~A}-7 \mathrm{C}$ suggest that there is an evolution from heulandite stability at temperatures $<20^{\circ} \mathrm{C}$ through stilbite stability and finally an apparent equilibrium between chabazite and stilbite at temperatures $>50^{\circ} \mathrm{C}$ (Site 793). Heulandite is a relatively uncommon component of recent sediments; according to Gottardi and Galli (1985), its surface genesis has never been documented. However, as pointed out by Kastner (1979), for example, the distinction between heulandite and clinoptilolite by XRD requires heat treatment of the samples, although this has not been routinely performed (and was not performed by the Leg 126 Shipboard Scientific Party). As for the $\mathrm{Na}_{2} \mathrm{O}-\mathrm{Al}_{2} \mathrm{O}_{3}-\mathrm{SiO}_{2}-\mathrm{H}_{2} \mathrm{O}$ diagrams, the most interesting feature of the $\mathrm{CaO}-\mathrm{Al}_{2} \mathrm{O}_{3}-\mathrm{SiO}_{2}-\mathrm{H}_{2} \mathrm{O}$ diagrams is the fact that the composition of the pore waters is close to equilibrium with several zeolite phases. For the same reasons as given above, this shows that chabazite, stilbite, and heulandite may act as sinks for $\mathrm{Ca}^{2+}$. Thus, one would not expect to observe systematic depth-related variations in the Ca-zeolite assemblage either.

The presence of wairakite in the Oligocene section at Site 792 raises a problem. At fixed $\mathrm{P}$ and $\mathrm{T}$, the relative stability of wairakite and chabazite depends only on the activity of water. With the thermodynamic parameters estimated here, wairakite becomes stable relative to chabazite only at temperatures $>250^{\circ} \mathrm{C}$. This is in agreement with observations reported by others (e.g., Coombs et al., 1959; Surdam and Boles, 1979). However, the abundance of smectites and scarcity of chlorites at Site 792 indicate that the sediments have never experienced temperatures this high. At present, the mode of genesis of wairakite at Site 792 is obscure. The Oligocene section was deposited by debris flow and turbidites, and the possibility that wairakite has been formed elsewhere cannot be excluded. This may also be true for any of the other phases, but in contrast, the pore-water data do not exclude a diagenetic origin of chabazite, stilbite, or heulandite.

\section{$\mathrm{K}_{2} \mathrm{O}-\mathrm{Al}_{2} \mathrm{O}_{3}-\mathrm{SiO}_{2}-\mathrm{H}_{2} \mathrm{O}$}

Declining $\mathrm{K}^{+}$concentrations with increasing depth is an ubiquitous feature of the pore waters of deep-sea sediments (e.g., Gieskes, 


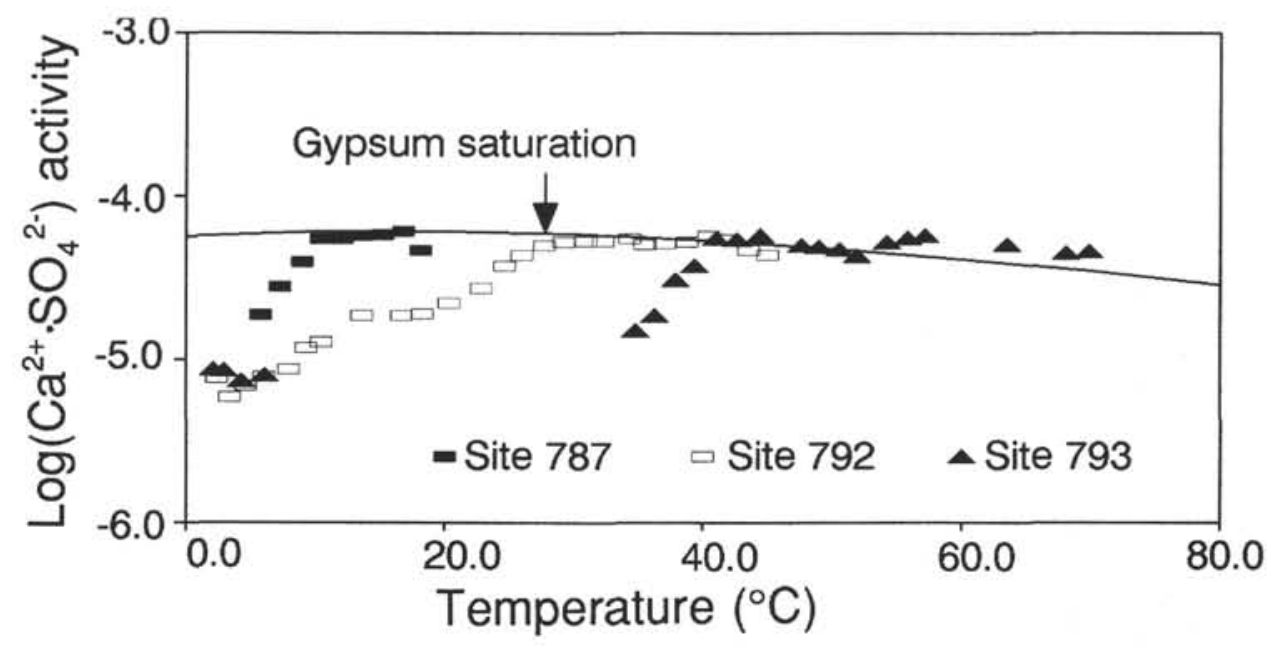

Figure 3. Comparison of the ion activity product of gypsum with the solubility of gypsum. The activity of water is close to 1.0 and is not included in the calculations.

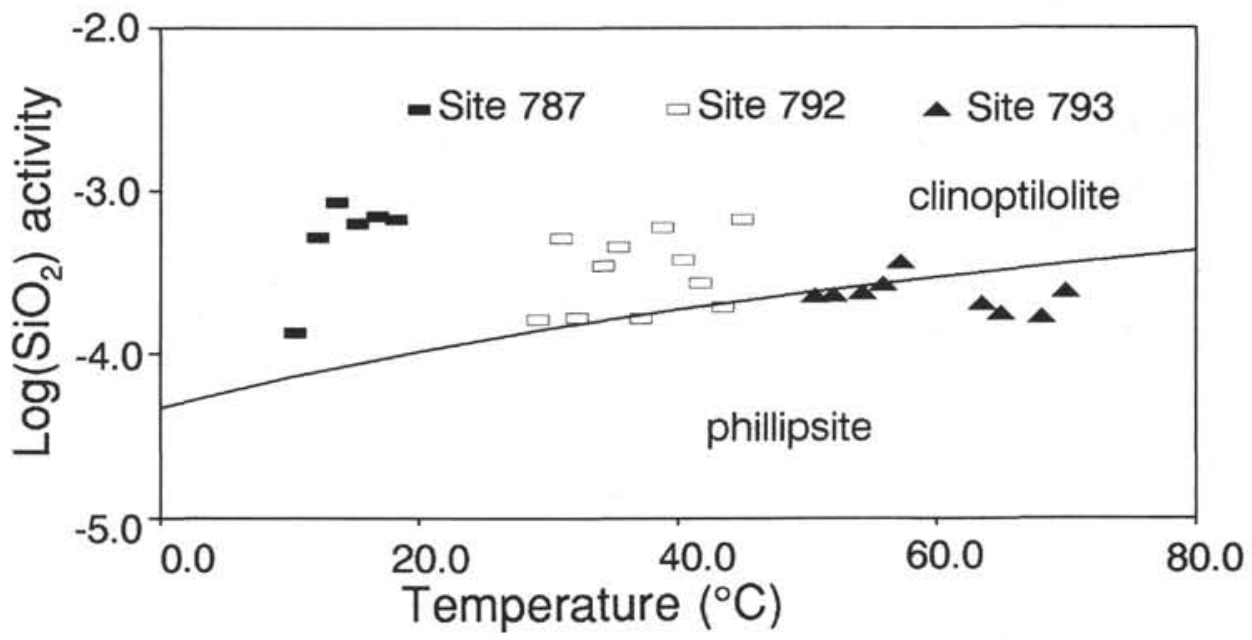

Figure 4. Relative stability of phillipsite and clinoptilolite.

1983). In the Oligocene sections at these sites, $\mathrm{K}^{+}$drops to very low values (Table 1). This suggests a sink for $\mathrm{K}$.

Compared with zeolites, $\mathrm{K}$-feldspar is rarely reported as an early authigenic phase of deep-sea sediments and was not detected in this study. Thus, although it is more stable than phillipsite and clinoptilolite, it has not been included in the stability diagrams (Figs. $8 \mathrm{~A}-8 \mathrm{C}$ ). The distribution of the data in these diagrams is in accordance with the frequently reported stability of clinoptilolite over phillipsite. Thus, the neoformation of clinoptilolite or the transformation of phillipsite into clinoptilolite (Boles and Wise, 1978) may take place in most samples at temperatures $<20^{\circ} \mathrm{C}$. At higher temperatures, the pore waters become undersaturated with respect to both zeolites. This suggests that at the corresponding depths, $\mathrm{K}^{+}$does not form separate phases but substitutes for other cations or is fixed in interlayer positions in smectites. Mixed-layer illite/smectite is a common authigenic component of argillaceous sediments buried to depths corresponding to temperatures of $50^{\circ} \mathrm{C}$ or more (e.g., Hower et al., 1976) and were found by shipboard XRD analyses of bulk rocks at Site 793. This constitute a potential sink for $\mathrm{K}^{+}$when the temperature exceeds about $50^{\circ} \mathrm{C}$ (Site 793).

\section{Summary of the Thermodynamic Analyses}

The results of the thermodynamic analyses are in general agreement with the mineralogical observations, which reveal the presence of numerous phases and no systematic zonation. All observed phases except wairakite, phillipsite, and chlorite could have precipitated at some point along the evolutionary path of the pore waters. Phillipsites are unstable at all depths and $\mathrm{K}$-clinoptilolites are stable up to $30^{\circ} \mathrm{C}$. The temperature range from $30^{\circ}$ to $50^{\circ} \mathrm{C}$ represents an interval in which only a small perturbation of the system can trigger precipitation of any of the phases: heulandite, Na-clinoptilolite, chabazite, analcime, stilbite, and trioctahedral smectites. At temperatures higher than $50^{\circ} \mathrm{C}$, stilbite, chabazite, and trioctahedral smectites constitute the most stable mineral assemblage. Illite in mixed-layer illite/smectites is the most probable sink of $\mathrm{K}^{+}$at temperatures exceeding $50^{\circ} \mathrm{C}$.

The pore-water concentrations of $\mathrm{Ca}^{2+}$ and $\mathrm{Na}^{+}$reach more or less stable levels at depth (Fig. 2). By assuming congruent dissolution and that the composition of the reacting material does not vary much throughout the Oligocene sections, the zeolite assemblage should become increasingly calcic with increasing depth. Qualitatively, this 
Table 2. End-member composition of zeolites identified by the shipboard X-ray diffraction analyses.

\begin{tabular}{|c|c|}
\hline Mineral & Composition \\
\hline $\begin{array}{l}\text { Wairakite } \\
\text { Heulandite } \\
\text { Chabasite } \\
\text { Stilbite } \\
\text { Analcime } \\
\text { Na-clinoptilolite } \\
\text { Na-phillipsite } \\
\text { K-clinoptilolite } \\
\text { K-phillipsite }\end{array}$ & 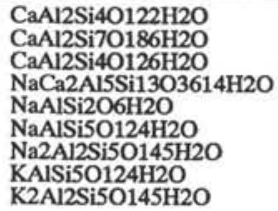 \\
\hline
\end{tabular}

agrees with the thermodynamic analyses and with the mineralogical observations (Taylor, Fujioka, et al., 1990). However, thermodynamics cannot be used quantitatively. For example, although stilbite and chabazite appear to be the most stable phases at high temperatures, they need only be present in trace amounts. This fact and the great number of thermodynamically permissible phases are major obstacles in comparisons of pore-water chemistry and mineralogy.

\section{Sites with Weak Concentration Gradients}

Despite the abundance of volcanogenic material in the upper sedimentological units (0-249 mbsf, Site 788; 0-165 mbsf, Site 790; 0-428 mbsf, Site 791), the pore-water concentration profiles at these sites are radically different from the concentration profiles at site 787 , 792 and 793 in that none of the major elements $\left(\mathrm{Na}^{+}, \mathrm{Mg}^{2+}, \mathrm{Ca}^{2+}\right.$, and $\mathrm{Cl}^{-}$) exhibit steep concentration gradients that can be attributed to the alteration of volcanogenic material.

The absence of steep concentration gradients at these sites, and the seawater-like composition of the pore water, may signify either low rates of reaction, a short lifetime of the system, or downward advection of seawater. Advection has two effects: (1) it increases the effective water/rock ratio, and (2) it alters the temperature distribution. The rate of dissolution of glass decreases with decreasing temperature and increasing $\mathrm{SiO}_{2}$ content of the glass (Fig. 9). The pumice samples from Sites 787, 790, and 791 analyzed by Thompson and Gill (this volume) exhibit a range of $\mathrm{SiO}_{2}$ from $49.09 \%$ to $75.53 \%$. To my knowledge, no data are available on the activation energies of the linear dissolution of rhyolitic glasses. Note, however, that, except for the data by Gislason and Eugster (1987), quartz, amorphous silica, tektites, and volcanic glasses exhibit a narrow range of activation energies (Fig. 9). The rate of dissolution of pumice is approximated by the data from White and Claassen (1980) with an activation energy of 65 kilo joule.

The lowest hypothetical rate of pumice dissolution is obtained when downward advection is sufficiently rapid to cool the sediments to the temperature of the bottom water $\left(\mathrm{ca} .2^{\circ} \mathrm{C}\right)$. However, given a specific surface area of the pumice of $5 \mathrm{~m}^{2} \mathrm{~g}^{-1}$ (Table 3), even at this lowest possible rate of dissolution, the entire pumice sections would dissolve in $<100 \mathrm{yr}$. Evidently, dissolution of pumice is not the rate-determining step. It is well known that precipitation of $\mathrm{SiO}_{2}$ in authigenic minerals controls the rate of low-temperature diagenetic transformation of silica phases (e.g., Kastner, 1981). The commonly observed supersaturation of pore waters with respect to silica phases can best be understood in terms of heterogeneous nucleation kinetics (e.g., Morse and Casey, 1988). Present theories for heterogeneous nucleation kinetics are not sufficiently advanced to be applied to natural sediments. I have taken a more pragmatic approach here. Opal-CT and quartz are the two most common authigenic silica phases in deep-sea sediments, with opal-CT usually forming first. Thus, opal-CT is the most likely sink of $\mathrm{SiO}_{2}$ released by dissolution of pumice. Based on a compilation of data from 37 DSDP volumes, Kastner (1979) established the relationship between temperature and time for the formation of opal-CT in deep-sea sediments.

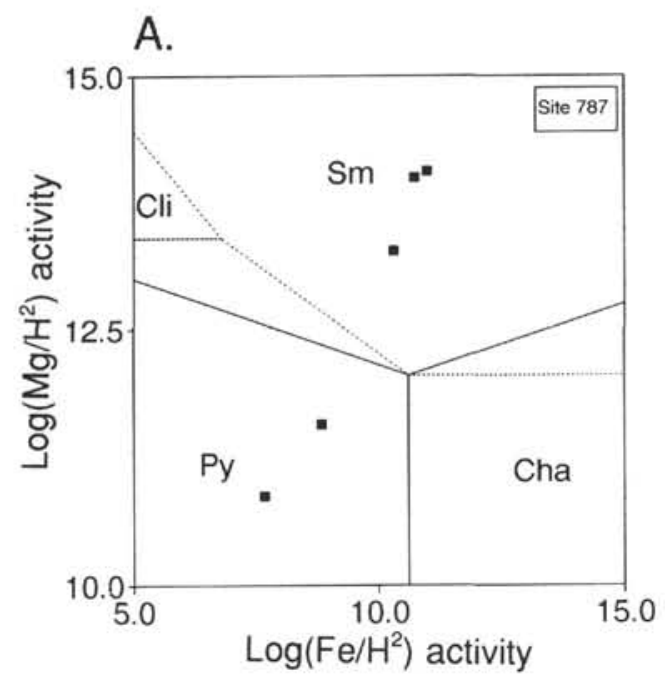

B.

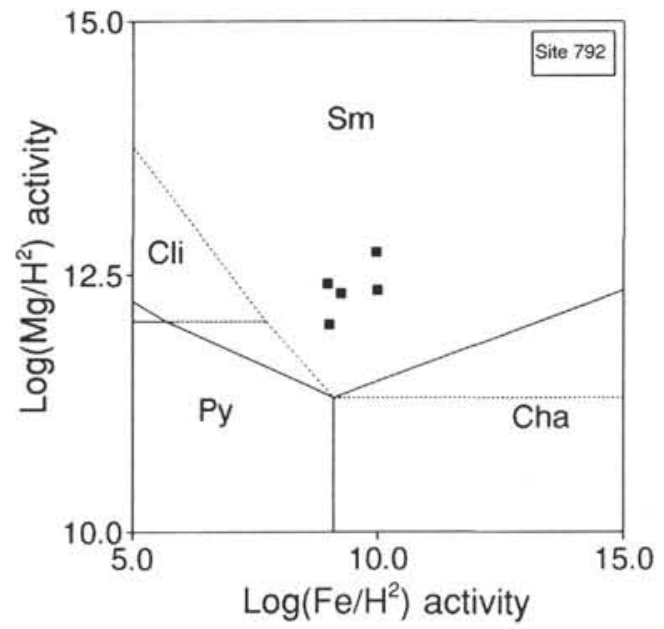

C.

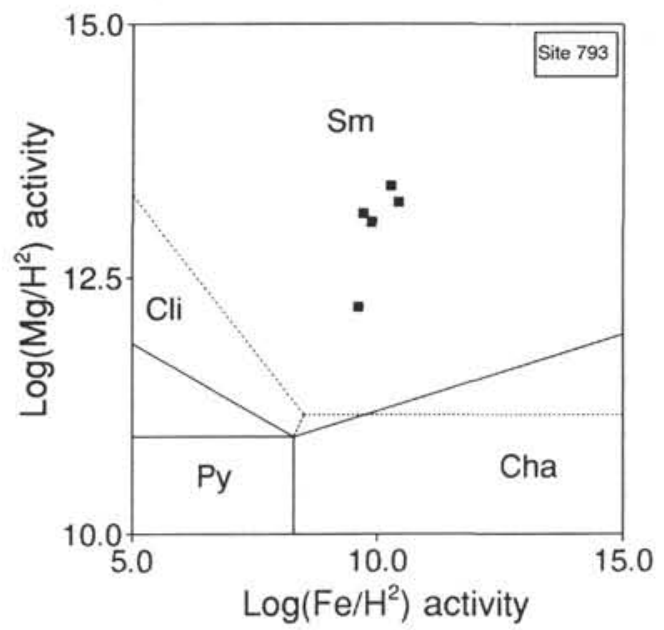

Figure 5. Data from Sites 787, 792, and 793 plotted on activity diagrams for the system $\mathrm{FeO}-\mathrm{MgO}-\mathrm{Al}_{2} \mathrm{O}_{3}-\mathrm{SiO}_{2}-\mathrm{H}_{2} \mathrm{O}$ at $15^{\circ}(\mathrm{A}), 40^{\circ}(\mathrm{B})$, and $60^{\circ} \mathrm{C}(\mathrm{C})$. Heavy and light lines represent the stability boundaries for trioctahedral smectite compositions: $\mathrm{Na}_{0.33}\left(\mathrm{Mg}_{2.55} \mathrm{Fe}_{0.45}\right)\left(\mathrm{Al}_{0.33} \mathrm{Si}_{3.67}\right) \mathrm{O}_{10}(\mathrm{OH})_{2}$, and $\mathrm{Na}_{0.33}\left(\mathrm{Mg}_{2.167} \mathrm{Fe}_{0.833}\right)\left(\mathrm{Al}_{0.33}\right.$ $\left.\mathrm{Si}_{3.67}\right) \mathrm{O}_{10}(\mathrm{OH})_{2}$, respectively. The phase boundaries of the smectites were estimated at the average $\mathrm{Na}^{+} / \mathrm{H}^{+}$activity ratio of each temperature interval. $\mathrm{Cli}=$ clinochlore, $\mathrm{Cha}=$ chamosite, $\mathrm{Sm}=$ trioctahedral smectite, and $\mathrm{Py}=$ pyrophyllite. 
A.

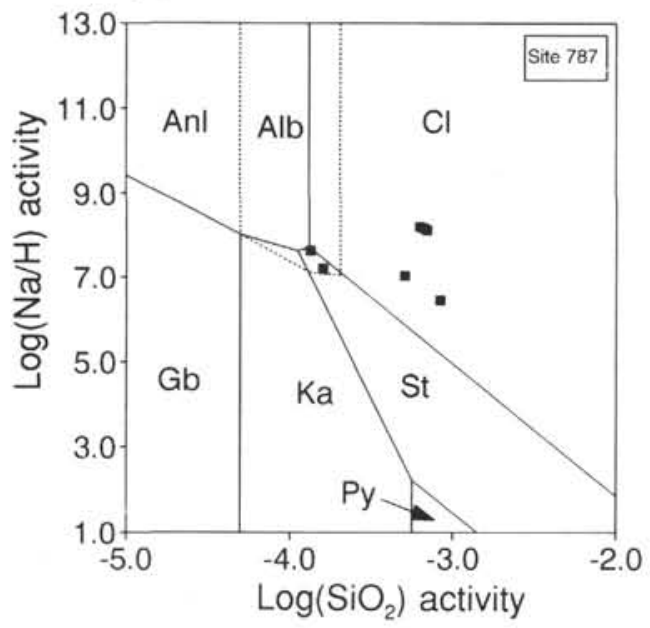

B.

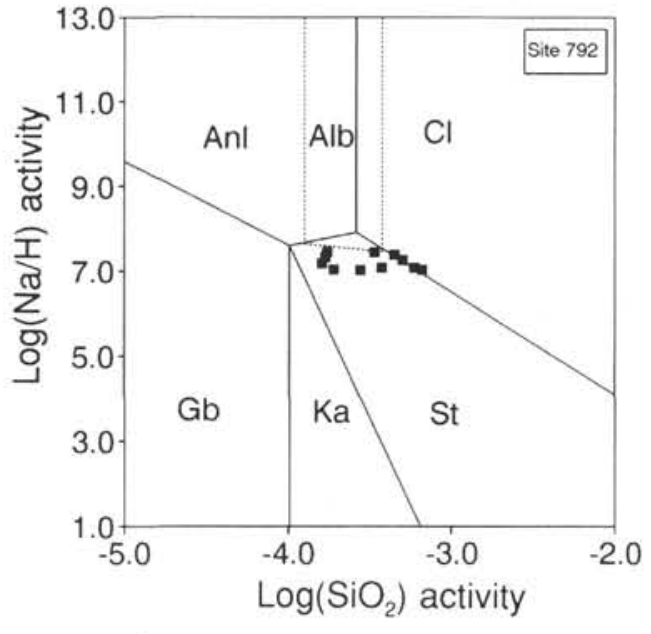

C.

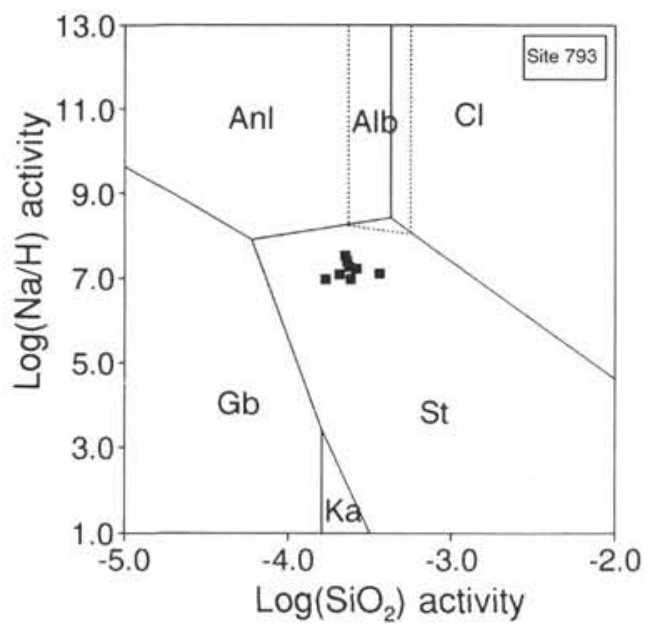

Figure 6. Data from Sites 787, 792, and 793 plotted on activity diagrams for the system $\mathrm{Na}_{2} \mathrm{O}-\mathrm{Al}_{2} \mathrm{O}_{3}-\mathrm{SiO}_{2}-\mathrm{H}_{2} \mathrm{O}$ at $15^{\circ}(\mathbf{A}), 40^{\circ}(\mathbf{B})$, and $60^{\circ} \mathrm{C}$ (C). The phase boundaries of stilbite were estimated at the average $\left[\mathrm{Ca}^{2+}\right] /\left[\mathrm{H}^{+}\right]^{2}$ activity ratio of each temperature interval. The broken line represents the stability field of albite (Alb). Anl = analcime, $\mathrm{Cl}=$ clinoptilolite, $\mathrm{St}=$ stilbite, $\mathrm{Gb}=$ gibbsite, $\mathrm{Ka}=$ kaolinite, and $\mathrm{Py}=$ pyrophyllite.
A.

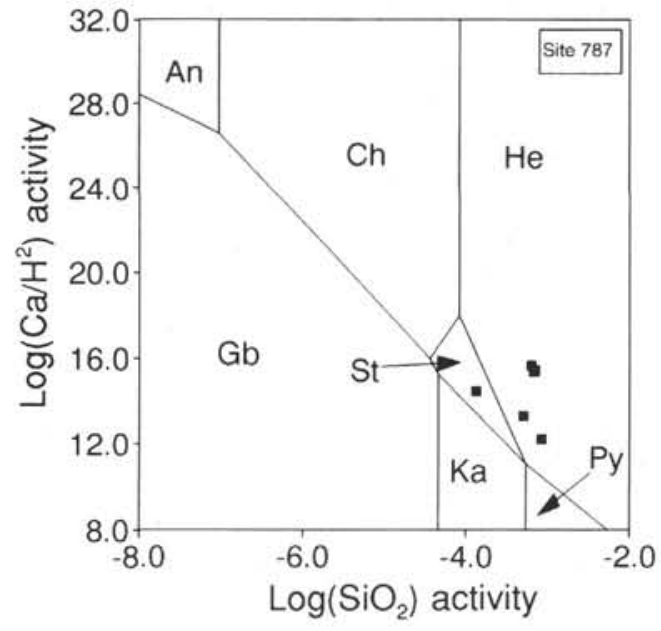

B.

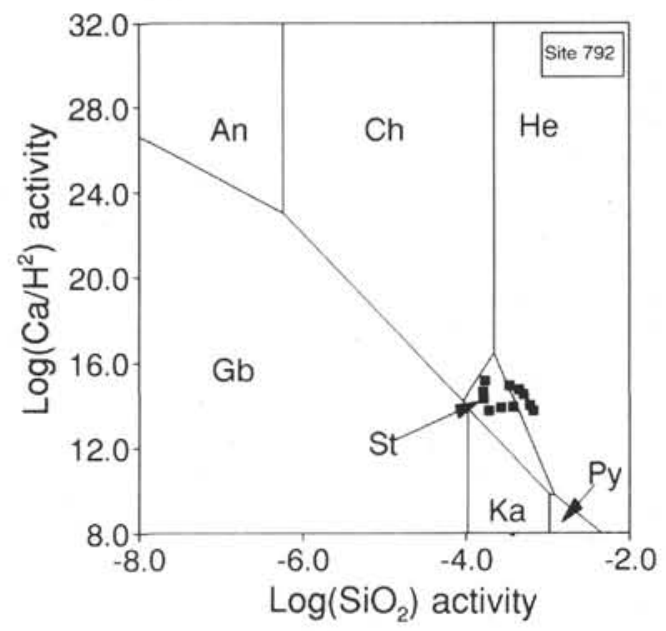

C.

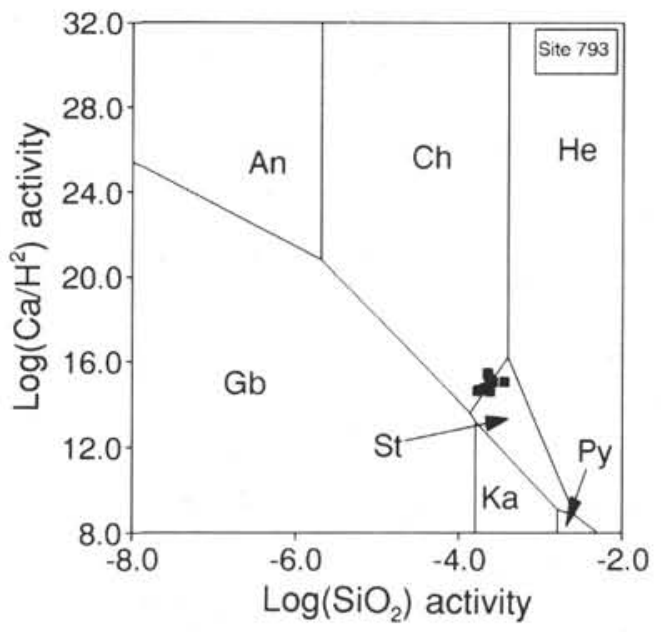

Figure 7. Data from Sites 787, 792, and 793 plotted on activity diagrams for the system $\mathrm{CaO}-\mathrm{Al}_{2} \mathrm{O}_{3}-\mathrm{SiO}_{2}-\mathrm{H}_{2} \mathrm{O}$ at $15^{\circ}(\mathbf{A}), 40^{\circ}(\mathbf{B})$, and $60^{\circ} \mathrm{C}(\mathbf{C})$. The phase boundaries of stilbite were estimated at the average $\mathrm{Na}^{+} / \mathrm{H}^{+}$activity ratio of each temperature interval. $\mathrm{An}=$ anorthite, $\mathrm{Ch}=$ chabazite, $\mathrm{He}=$ heulandite, $\mathrm{St}=$ stilbite, $\mathrm{Gb}=$ gibbsite, $\mathrm{Ka}=$ kaolinite, and $\mathrm{Py}=$ pyrophyllite . 
The major problem in applying this relationship to Sites 787, 790, and 791 is the lack of downhole temperature measurements at these backarc sites. However, a first-order estimate of the thermal gradients may be obtained from heat flow measurements. Matsubara (1981) and Yamazaki (1988) attributed the large range of heat-flow values (54 $700 \mathrm{~mW} / \mathrm{m}^{2}$ ) in the backarc region of the Izu-Ogasawara arc to hydrothermal activity. The frequency distribution of their data shows a distinct peak at about $100 \mathrm{~mW} / \mathrm{m}^{2}$ indicating that this is the "normal" value in the absence of advection. With a bulk-sediment, thermal conductivity value of $0.9 \mathrm{~W} /(\mathrm{m} \bullet \mathrm{K})$ (Taylor, Fujioka, et al., 1990), this is equivalent to a thermal gradient of $111^{\circ} \mathrm{C} \bullet \mathrm{km}^{-1}$.

The reason for the contrasting pore-water chemistry between those sites with steep concentration gradients $(787,792$, and 793$)$ and those sites with weak concentration gradients $(788,790$, and 791$)$ is indicated by Figure 10. Although the thermal gradients are twice as high as at the sites with steep concentration gradients, the pumiceous sections are too young and too cool to allow precipitation of opal-CT. Hence, once the pore waters have equilibrated with the pumice, there is no driving force for continued reaction. Note that at Site 787 about $1 \mathrm{~km}$ of the section has been truncated by erosion; thus, although Figure 10 indicates otherwise, the conditions for opal-CT have been met at this site.

The arguments presented above show that the absence of diagenetic imprints on the composition of the pore waters at Sites 788, 790, and 791 can be understood without invoking hydrothermal advection. The diagenetic "quiet zone" is not caused by low reactivity of the sedimentary components but, rather, by the slow nucleation of silicaremoving reactions. Note, however, that these arguments do not exclude the possibility that downward advection is taking place.

\section{CONCLUSIONS}

The sites examined in this study may be classified into two categories depending on the presence (Group I: Sites 787, 792, and 793) or absence (Group II: Sites 788, 790, and 791) of steep concentration gradients. A hypothesis that these groups represent reactiondominated and advection-dominated sites respectively was tested.

It was found that the absence of steep concentration gradients at the Group II sites can be explained without invoking downwelling of seawater. The overall rate of transformation of the pumiceous sections at these sites is controlled by the rate of precipitation of $\mathrm{SiO}_{2}$. The rate of precipitation of $\mathrm{SiO}_{2}$ is probably governed by the slow heterogeneous nucleation kinetics of opal-CT.

The highly altered composition of the pore waters in the volcanic Oligocene sections at the Group I sites suggests that a number of diagenetic minerals may have precipitated during the evolution of these waters. This hypothesis was explored by comparing the activities of dissolved species with the thermodynamic stability of selected observed diagenetic minerals (zeolites, chlorites, smectites, micas, feldspars, and gypsum).

At all sites, the pore waters reach equilibrium with respect to gypsum, but gypsum is not a major sink of $\mathrm{Ca}^{2+}$. All observed phases except wairakite, chlorite, and phillipsite could have precipitated at some point along the evolutionary path of the pore waters. Phillipsites are unstable at all depths, and $\mathrm{K}$-clinoptilolites are stable up to $30^{\circ} \mathrm{C}$. The temperature range from $30^{\circ}$ to $50^{\circ} \mathrm{C}$ represents an interval in which only a small perturbation of the system can trigger precipitation of any of the phases: heulandite, Na-clinoptilolite, chabazite, analcime, stilbite, and trioctahedral smectites. At temperatures $>50^{\circ} \mathrm{C}$, stilbite, chabazite, and trioctahedral smectites are stable. Illite in mixed-layer illite/smectites is the most probable sink of $\mathrm{K}^{+}$at temperatures exceeding $50^{\circ} \mathrm{C}$.

The absence of systematic vertical zonations in the authigenic mineral assemblages may be understood in terms of the large number of thermodynamically permissible phases and the slow rate of the dissolution of authigenic minerals.
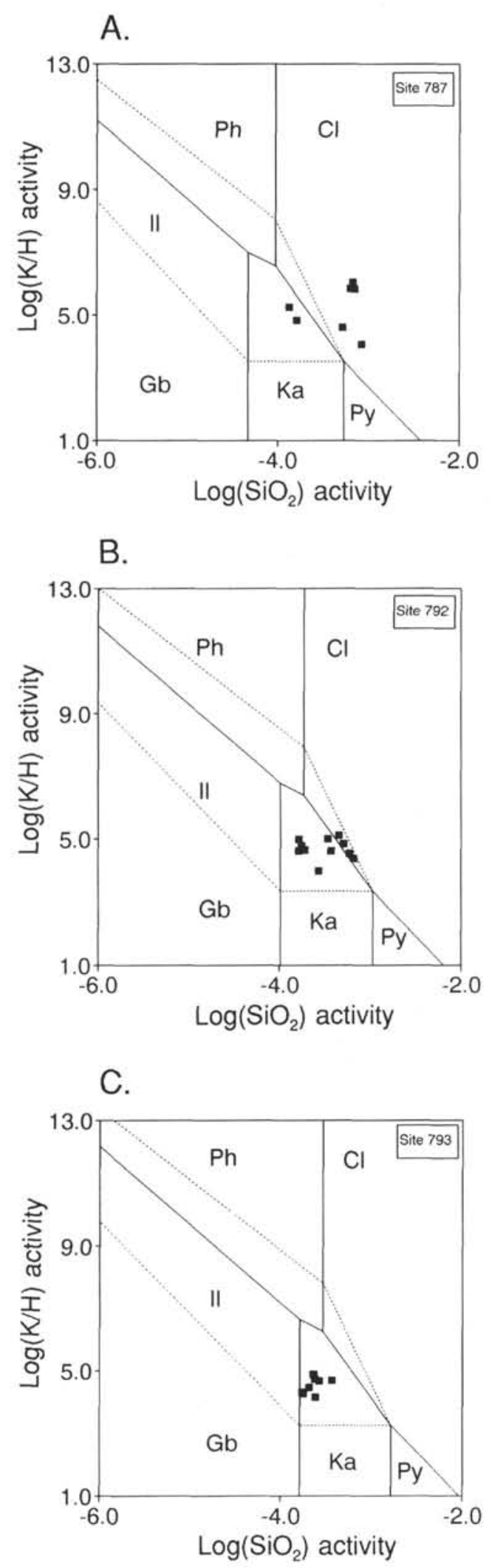

Figure 8. Data from Sites 787, 792, and 793 plotted on activity diagrams for the system $\mathrm{K}_{2} \mathrm{O}-\mathrm{Al}_{2} \mathrm{O}_{3}-\mathrm{SiO}_{2}-\mathrm{H}_{2} \mathrm{O}$ at $15^{\circ}(\mathrm{A}), 40^{\circ}(\mathrm{B})$, and $60^{\circ} \mathrm{C}(\mathrm{C})$. The broken line represents the stability field of illite (II). $\mathrm{Ph}=$ phillipsite, $\mathrm{Cl}=$ clinoptilolite, $\mathrm{Gb}=$ gibbsite, $\mathrm{Ka}=$ kaolinite, and $\mathrm{Py}=$ pyrophyllite. 


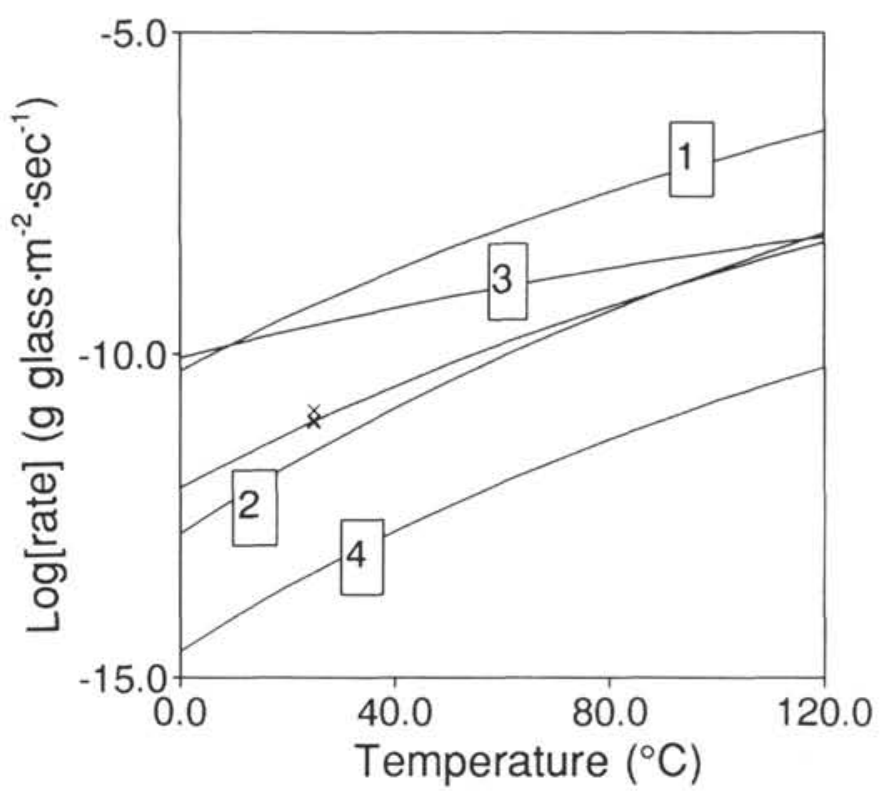

Figure 9. Rate of dissolution of various silica phases. Line $1=$ tholeiitic glass (Crovisier et al., 1985), line 2 = tektites (Barkatt et al., 1984), line $3=$ basaltic glass (Gislason and Eugster, 1987), line 4 = quartz (Rimstidt and Barnes, 1980). Data points (shown by $\mathrm{X})=$ rhyolitic glasses (White and Claassen, 1980).

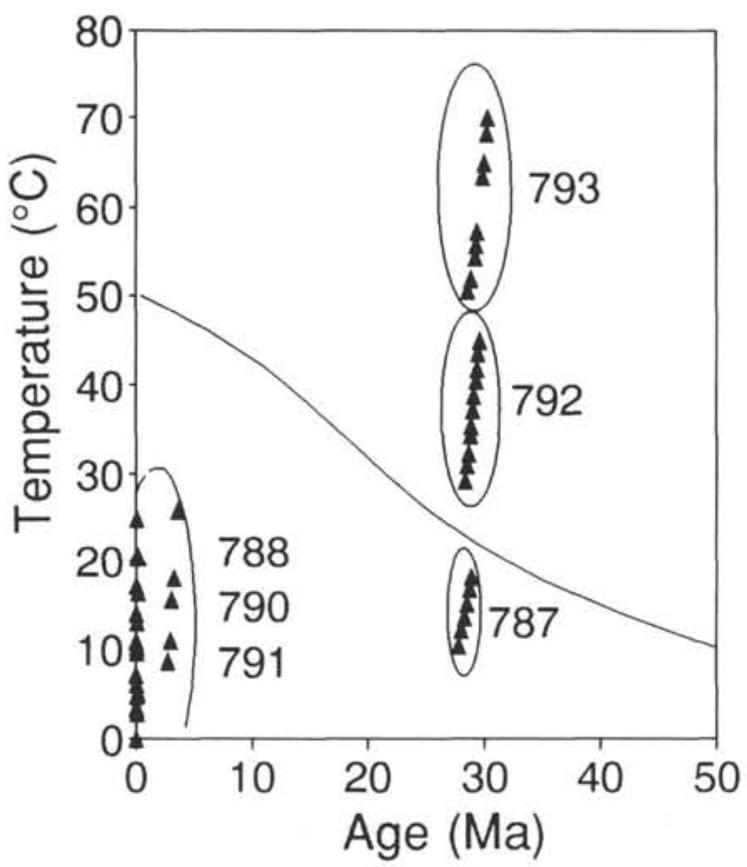

Figure 10. The temperature and age of the sediments from the pumiceous sections (Sites 788, 790, and 791) and the Oligocene volcaniclastic sections (Sites 787, 792, and 793) compared with the empirical line for opal-CT transformation from Kastner (1979).
Table 3. Specific surface area of pumice fragments compared with the specific surface area of quartz sand.

\begin{tabular}{lcc}
\hline Mineral & $\begin{array}{c}\text { Size } \\
\text { range } \\
(\mu \mathrm{m})\end{array}$ & $\begin{array}{c}\text { Surface } \\
\text { area } \\
(\mathrm{m} 2 \mathrm{~g}-1)\end{array}$ \\
\hline Pumice & $<100$ & 4.8 \\
Pumice & $100-250$ & 7.1 \\
Pumice & $250-1000$ & 5.1 \\
Pumice & $1000-2000$ & 8.3 \\
Pumice & $2000-5000$ & 8.1 \\
Quartz & $250-1000$ & 0.1 \\
\hline Notes: Steen-Mclntyre (1975) advo-
\end{tabular}
cated the use of the hydration rate of pumice as a means of dating ash beds. She found that, for particles $>62-\mu \mathrm{m}$, the particle size of pumice fragments has little effect on the rate of hydration. This may be taken as an indication that because of its vesicular nature the specific surface area of pumice reaches a constant level for fragments $>62$ $\mu \mathrm{m}$. The results of the single point $\mathrm{N} 2$-adsorption surface determinations show that the specific surface area of pumice is about 1.5 orders of magnitude larger than for quartz sand, and that indeed the specific surface area is almost independent of particle size for particles between about 100 and $5000 \mu \mathrm{m}$.

\section{ACKNOWLEDGMENTS}

This study was funded by the Norwegian Research Council for Science and the Humanities (NAVF). I would also like to thank the Leg 126 technical staff and especially J. Perry and V. Clark.

\section{REFERENCES}

Aagaard, P., and Helgeson, H. C., 1983. Activity/composition relations among silicates and aqueous solutions: II. Chemical and thermodynamic consequences of ideal mixing of atoms on homological sites in montmorillonites, illites and mixed-layer clays. Clays Clay Miner., 31:207-218.

Barkatt, A., Boulos, M. S., Barkatt, A., Sousanpour, W., Boroomand, M. A., Macedo, P. B., and O'Kefee, J. A., 1984. The chemical durability of tektites; a laboratory study of long-term corrosion behaviour. Geochim. Cosmochim. Acta, 48:361-371.

Boles, J. R., 1981a. Zeolites in low-grade metamorphic rocks. In Mumpton, F. A. (Ed.), Mineralogy and Geology of Natural Zeolites. Mineral. Soc. Am., Rev. Mineral., 4:103-135.

, 1981b. Zeolites in deep-sea sediments. In Mumpton, F. A. (Ed.), Mineralogy and Geology of Natural Zeolites. Rev. Mineral., 4:137-163.

Boles, J. R., and Wise, W. S., 1978. Nature and origin of deep sea clinoptilolite. In Sand, L. B., and Mumpton, F. A. (Eds.), Natural Zeolites: Occurrence, Properties, Use: Elmsford, NY (Pergamon Press), 235-244.

Coombs, D. S., 1954. The nature and alteration of some Triassic sediments from Southland, New Zealand. Trans. R. Soc. N. Z., 82:65-109.

Coombs, D. S., Ellis, A. J., Fyfe, W. S., and Taylor, A. M., 1959. The zeolite facies, with comments on the interpretation of hydrothermal syntheses. Geochim. Cosmochim. Acta, 17:53-107.

Cosgrove, M. E., and Papavassiliou, C. T., 1979. Clinoptilolite in DSDP sediments of the Indian Ocean (Site 223, Leg 23): its stability conditions and estimation of its free energy. Mar. Geol., 33:M77-M84. 
Crovisier, J. L., Fritz, B., Grambow, B., and Eberhart, J. P., 1985. Dissolution of basaltic glass: experiments and thermodynamic modelling. In Werme, L. (Ed.), Scientific Basis for Nuclear Waste Management. M.R.S. Symp. Proc., 50:273-280.

Egeberg, P. K., and Leg 126 Shipboard Scientific Party, 1990. Unusual composition of pore waters found in the Izu-Bonin fore-arc sedimentary basin. Nature, 344:215-218.

Gieskes, J. M., 1983. The chemistry of interstitial waters of deep-sea sediments: interpretation of Deep-Sea Drilling data. In Riley, J. P., and Chester, R. (Eds.), Chemical Oceanography (Vol. 8): London (Academic Press), 222-269.

Gislason, S. R., and Eugster, H. P., 1987. Meteoric water-basalt interactions. I: A laboratory study. Geochim. Cosmochim. Acta, 51:2827-2840.

Gottardi, G., and Galli, E., 1985. Natural Zeolites: Berlin (Springer-Verlag).

Helgeson, H. C., Delany, J. M., Nesbitt, H. W., and Bird, D. K., 1978. Summary and critique of the thermodynamic properties of rock-forming minerals. Am. J. Sci., 278A.

Hower, J., Eslinger, E. V., Hower, M. E., and Perry, E. A., 1976. Mechanism of burial metamorphism of argillaceous sediment: mineralogical and chemical evidence. Geol. Soc. Am. Bull., 87:725-737.

Iglesia, A. La, and Aznar, A. J., 1986. A method of estimating the Gibbs energies of formation of zeolites. Zeolites, 6:26-29.

Kastner, M., 1979. Zeolites. In Burns, R. G. (Ed.), Marine Minerals. Rev. Mineral., 6:111-122.

, 1981. Authigenic silicates in deep sea sediments: formation and diagenesis. In Emiliani, C. (Ed.), The Sea (Vol. 7): New York (Wiley), 915-980.

Kharaka, Y. K., Gunter, W. D., Aggarwall, P. K., Perkins, E. H., and DeBraal, J. D., 1988. SOLMINEQ: a computer program for geochemical modelling of water rock interactions. Invest. Rep. Wat. Resour. U.S. Geol. Surv., No. 88-4277.

MacEvan, D.M.C., and Wilson, M. J., 1980. Interlayer and intercalation complexes of clay minerals. In Brindley, G. W., and Brown, G. (Eds.), Crystal Structures of Clay Minerals and Their X-ray Identification. London Mineral. Soc., 197-248.

Manheim, F. T., and Sayles, F. L., 1974. Composition and origin of interstitial waters of marine sediments based on deep sea drill cores. In Goldberg, E. D. (Ed.), The Sea (Vol. 5): New York (Wiley Interscience), 527-568.
Manheim, F. T., and Sayles, F. L., 1974. Composition and origin of interstitial waters of marine sediments based on deep sea drill cores. In Goldberg, E. D. (Ed.), The Sea (Vol. 5): Marine Chemistry: New York (Wiley Interscience), 572-568.

Matsubara, Y., 1981. Heat flow measurements in the Bonin Arc area. In Honza, E., Inoue, E., and Ishihara, T. (Eds.), Geological Investigation of the Ogasawara (Bonin) and Northern Mariana Arcs. Cruise Rep., Geol. Surv. Jpn., 14:130-136.

Morse, J. W., and Casey, W. H., 1988. Ostwald processes and mineral paragenesis in sediments. Am. J. Sci., 288:537-560.

Rimstidt, J. D., and Barnes, H. L., 1980. The kinetics of silica-water reactions. Geochim. Cosmochim. Acta, 44:1683-1699.

Steen-McIntyre, V., 1975. Hydration and superhydration of tephra glass: a potential tool for estimating age of Holocene and Pleistocene ash beds. In Suggate, R. P., and Cresswell, M. M. (Eds.), Quaternary Studies. Bull. R. Soc. N.Z., 13:271-278.

Stonecipher, S. A., 1977. Origin, distribution and diagenesis of deep-sea phillipsite and clinoptilolite [Ph.D. dissert.]. Univ. of California, San Diego.

Surdam, R. C., and Boles, J. R., 1979. Diagenesis of volcanic sandstones. In Scholle, P. A., and Schluger, P. R. (Eds.), Aspects of Diagenesis. Spec. Publ.-Soc. Econ. Paleontol. Mineral., 26:227-242.

Taylor, B., Fujioka, K., et al., 1990. Proc. ODP, Init. Repts., 126: College Station, TX (Ocean Drilling Program).

Walshe, J. L., 1986. A six-component chlorite solid solution model and the conditions of chlorite formation in hydrothermal and geothermal systems. Econ. Geol., 81:681-703.

White, A. F., and Claassen, H. C., 1980. Kinetic model for the short-term dissolution of a rhyolitic glass. Chem. Geol., 28:91-109.

Wolery, T. J., 1979. Calculation of chemical equilibrium between aqueous solution and mineral: the EQ3/EQ6 software package. Lawrence Livermore Laboratory, Univ. of California, Rep. URCL-52658.

Yamazaki, A. N., 1988. Heat flow in the Sumisu Rift, Izu-Ogasawara (Bonin) Arc. Bull. Geol. Soc. Jpn., 39:63-70.

Date of initial receipt: 15 November 1990

Date of acceptance: 30 April 1991

Ms 126B-153 